\title{
Application of APES to Adaptive Arrays on the CDMA Reverse Channel
}

\author{
David J. Russell and Robert D. Palmer, Senior Member, IEEE
}

\begin{abstract}
This work compares the performance of various beamforming algorithms for the mitigation of multiple access interference in adaptive array code-division multiple-access (CDMA) systems. The Fourier and linear constrained minimum variance (LCMV) methods are both well-known algorithms for beamforming. While the LCMV method has many advantages, it can result in high noise gain under certain conditions. The recently developed amplitude and phase-estimation (APES) method has been shown to have better noise-gain performance. The APES filter is derived for the spatial case and simulation results are compared to those of both Fourier and LCMV under various scenarios of the CDMA reverse channel. Further, the effects of the use of multiple constraints on the beam pattern of LCMV and APES are investigated.
\end{abstract}

Index Terms-Adaptive arrays, code-division multiple access (CDMA), multiaccess communication.

\section{INTRODUCTION}

$\mathbf{I}$ T IS well known [1] that one of the primary limitations of code-division multiple-access (CDMA) reverse-channel performance is multiple access interference (MAI). That is, because all subscribers for a single cell asynchronously transmit using the same carrier frequency, there exists mutual interference. For synchronous CDMA, each transmitted signal is spread by one of many mutually orthogonal codes, such as Walsh codes, and then despread by the receiver. However, when the transmitted signals are not synchronized with respect to the code sequences, the orthogonal nature of the codes is lost and other codes must be used, which have low cross-correlation characteristics, such as pseudonoise (PN) sequences.

Note that the forward channel does not normally suffer from MAI due to the fact that the base station (BS) broadcasts a composite signal and has control over the timing of the spreading sequences. Of course, channel effects, such as multipath, can degrade the orthogonality even in the forward channel. Nevertheless, the more challenging problems exist for the reverse channel and are the subject of much study.

For the case of a binary phase-shift keying (BPSK) digitalcommunication CDMA system and assuming that the BS has delay and phase synchronization with subscriber zero, the re-

Manuscript received February 12, 2002; revised August 27, 2002, January 7 , 2003, June 20, 2003, and September 26, 2003. This work was supported in part by EFJohnson Corporation, Lincoln, NE.

D. J. Russell is with EFJohnson, Lincoln, NE 68521-4429 USA (e-mail: drussell@efjohnson.com).

R. D. Palmer is with the Department of Electrical Engineering, University of Nebraska-Lincoln, Lincoln, NE 68588-0511 USA (e-mail bpalmer@unl.edu).

Digital Object Identifier 10.1109/TVT.2003.821991 verse channel decision statistic is composed of an estimate of the information bit from the signal of interest (SOI), the signals from all other users (MAI), multipath signals, and additive white Gaussian noise (AWGN) [1]. The MAI contribution to the decision statistic can simply be considered another noise source, with a colored spectrum [1]. Thus, it is desirable to mitigate the effects of MAI when detecting the SOI. Methods based on minimum mean-squared error (MMSE) and interference cancellation (IC) have had some success for this problem and have been studied in detail (see [2]-[5] and references therein). A complementary technique for reducing MAI is based on the use of adaptive arrays or smart antennas at the BS (see [1], [6], and [7] for an overview). This work focuses on competing techniques for the implementation of smart antenna technology in order to improve reverse channel performance.

\section{CONVENTIONAL BEAmFORMER (FOURIER)}

In general, an antenna array used in beamforming is made up of a fixed number of elements positioned with a specific geometry. For example, a uniform linear array (ULA) is quite common. Referring to Fig. 1, for an $M$-element array, the received signals are downconverted to baseband, processed with a pulse-shaped matched filter, and appropriately sampled, resulting in the received data vector defined as

$$
\mathbf{u}_{i}=\left[u_{0, i} \cdots u_{M-1, i}\right]^{T}
$$

where $i$ represents the $i$ th chip. Each element of the array receives a systematically modified version of the signal incident on the array. The signal consists of the sum of independently spread information sequences from each user, where each information sequence is randomly delayed and composed of random symbols. In addition, each receiver adds independent AWGN to the signal. Therefore, the signals from each receiver may be assumed to be stationary random processes. By exploiting the spatial diversity in the signals, a properly designed receiver is capable of coherently combining the signals from the multiple elements, resulting in an increased signal-to-noise ratio (SNR).

Individual CDMA receivers could be implemented on each element of the array. However, we will approach the problem using blind beamforming where no information about the SOI will be used until after beamforming. This approach simplifies the overall receiver design. The problem then becomes how to effectively combine the signals from the array. One typical solution is to linearly sum the signals with weighting defined by the vector

$$
\mathbf{w}=\left[w_{0} \cdots w_{M-1}\right]^{H}
$$




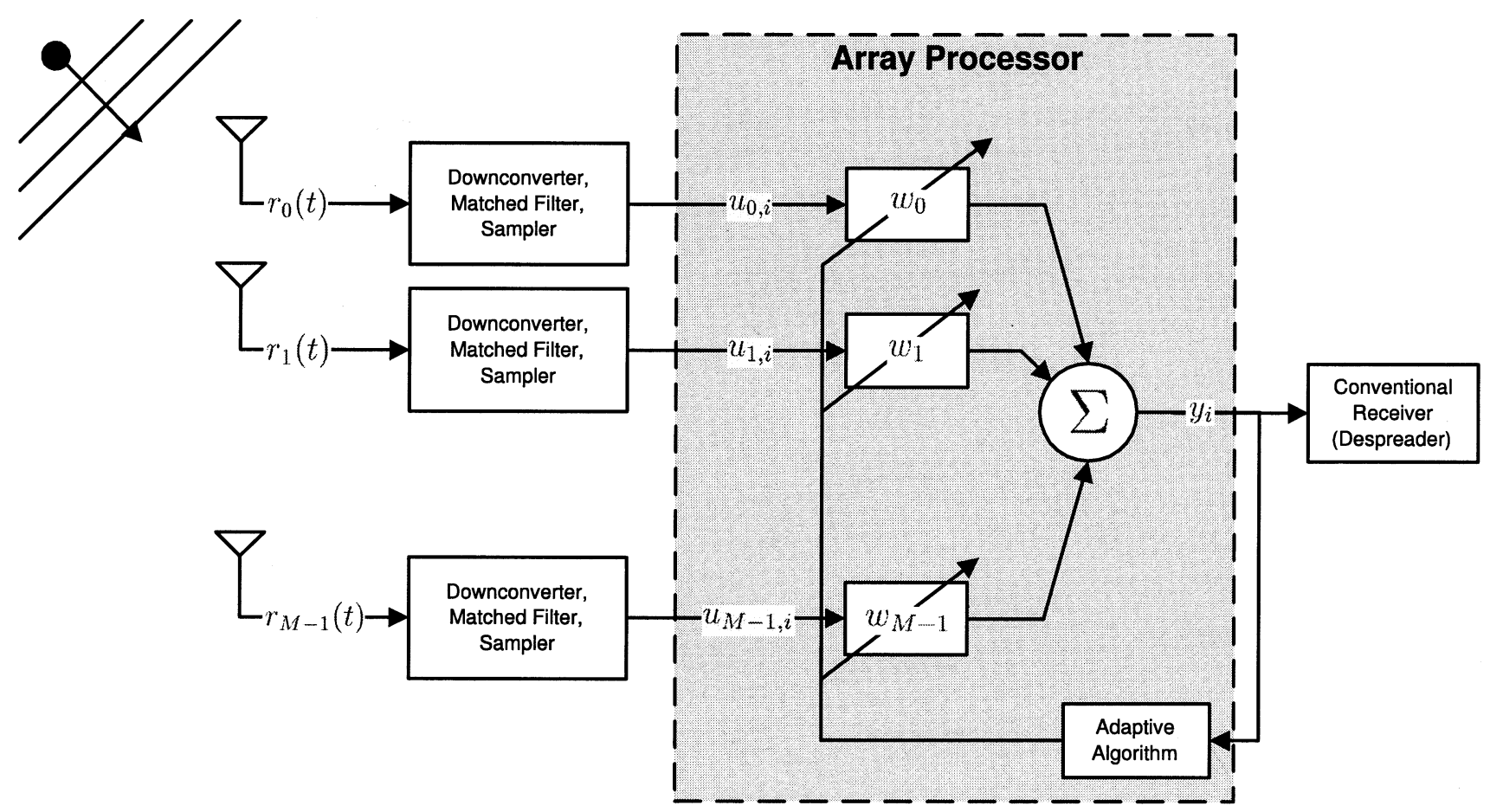

Fig. 1. Array processor filters $M$ corresponding digital signals with appropriately chosen complex weights and combines the outputs into a signal that is sent to the conventional receiver, i.e., despreader. In an adaptive system, the output is analyzed in order to adjust the weights for successive filtering.

in which the elements define complex weights that are applied to the respective elements of the array. By applying (2) to (1), the array output is given by

$$
y_{i}=\mathbf{w}^{H} \mathbf{u}_{i}
$$

which is eventually used to produce the decision statistic for a particular subscriber.

If (2) is viewed as a finite impulse response (FIR) spatial filter, it is obvious that the weights should be chosen to produce a high gain in the direction of arrival (DOA) of the SOI and low gains elsewhere.

One of the most intuitive methods of selecting the weight vector in (2) is the Fourier beamformer. Given that the SOI (subscriber zero) is incident on the array from elevation angle $\theta_{0}$ and azimuth angle $\phi_{0}$, the noise-free data vector would be $\mathbf{u}_{i}=\mathbf{a}_{0} d_{i, 0}$, where $d_{i, 0}$ is the spread information signal from subscriber zero and

$$
\mathbf{a}_{0}=\left[a_{0}\left(\theta_{0}, \phi_{0}\right) \cdots a_{M-1}\left(\theta_{0}, \phi_{0}\right)\right]^{T}
$$

is the steering vector directed toward $\left(\theta_{0}, \phi_{0}\right)$ based on an $M$-element array. The $m$ th component of the steering vector is given by

$$
a_{m}(\theta, \phi)=e^{-j \beta\left(x_{m} \cos \phi \sin \theta+y_{m} \sin \phi \sin \theta+z_{m} \cos \theta\right)}
$$

where the phase propagation factor is $\beta=\omega_{c} / c$ in which $\omega_{c}$ is the carrier frequency, $c$ is the speed of light $\left(3 \times 10^{8} \mathrm{~m} / \mathrm{s}\right)$, and $\left[x_{m}, y_{m}, z_{m}\right]$ are the Cartesian coordinates of element $m$ of the array.
If the weight vector was chosen as $\mathbf{w}_{F}=\mathbf{a}_{0}$, the resulting array output would be

$$
\begin{aligned}
y_{i} & =\mathbf{w}_{F}^{H} \mathbf{u}_{i} \\
& =\mathbf{a}_{0}^{H} \mathbf{a}_{0} d_{i, 0}
\end{aligned}
$$

which maximizes the gain in the direction of $\left(\theta_{0}, \phi_{0}\right)$ and is proportional to $d_{i, 0}$ with the addition of AWGN, MAI, and interchip interference. Thus, the Fourier beamformer does not explicitly attempt to attenuate noise or interference and the performance is, therefore, degraded with respect to the signal-to-interference-noise ratio (SINR).

\section{ADAPTIVE BEAMFORMING}

Unlike conventional beamforming, adaptive methods exploit the knowledge of the signals that exist within the field of view of the array. Therefore, adaptive beamforming can be used to attenuate noise and MAI while simultaneously maintaining the gain toward the SOI. Referring to Fig. 1, adaptive methods operate within the adaptive algorithm block in order to dynamically update each weight value.

Before attempting to derive an optimal weight vector, several important definitions are needed. The following vector represents a partition of elements from the data vector $\mathbf{u}_{i}$

$$
\mathbf{u}_{l, i}=\left[u_{l, i} \cdots u_{l+M^{\prime}-1, i}\right]^{T} \quad 0 \leq l<L
$$

where $L=M-M^{\prime}+1$ and $M^{\prime}$ is the number of array elements used in the spatial filter. Note that when $M^{\prime}<M$, the spatial filter uses fewer elements than those present in the array and angular resolution will suffer. As will be seen, the choice of 
$M^{\prime}<M$ allows the benefits of spatial smoothing, including sidelobe reduction, and will be discussed later in this section.

The partitioned data vectors may be organized into the $\left(M^{\prime} \times\right.$ $N)$ matrix

$$
\mathbf{U}_{l}=\left[\mathbf{u}_{l, 0} \cdots \mathbf{u}_{l, N-1}\right]
$$

where $N$ is the number of chips used by the adaptive algorithm and typically encompasses many symbols.

Assuming ergodicity [1], the spatial covariance matrix can be estimated using the temporal average equation

$$
\hat{\mathbf{R}}_{l}=\frac{1}{N} \mathbf{U}_{l} \mathbf{U}_{l}^{H}
$$

Given that there exists $L$ possible estimates of the spatial covariance matrix, the standard procedure is to average over all estimates

$$
\hat{\mathbf{R}}_{M^{\prime}}=\frac{1}{L} \sum_{l=0}^{L-1} \hat{\mathbf{R}}_{l} .
$$

However, by averaging the estimates in this manner, it is implied that the antenna array is restricted to a ULA geometry.

In their standard form, the following two adaptive methods constrain the optimization procedure to maintain unity gain toward the SOI. By exploiting knowledge of the other subscribers' DOAs in the cell, it may be possible to significantly reduce MAI. Multiple constraints can be added to the optimization procedure whereby a null in the antenna pattern is specifically designed into the array weights. These additional constraints are formalized by defining the constraint vector of length $\kappa$, corresponding to $\kappa$ constraint gains [8]

$$
\mathbf{c}=\left[c_{0} \cdots c_{\kappa-1}\right]
$$

where $c_{k}$ is the gain directed toward subscriber $k$. In most situations, $c_{0}$ is unity and all other gains are chosen to be zero.

Finally, define the steering matrix as

$$
\mathbf{A}=\left[\mathbf{a}_{0} \cdots \mathbf{a}_{\kappa-1}\right]
$$

which is constructed from multiple steering vectors corresponding to each of the constraints in (11).

\section{A. Linearly Constrained Minimum Variance (LCMV)}

The LCMV beamformer was first introduced by Capon [9]. This method is designed to minimize the output power of an FIR spatial filter while simultaneously maintaining unity gain toward some desired direction. In the present case, the desired direction will be toward the SOI.

In the standard spatial implementation of the LCMV method, the full array is used for beamforming with $M^{\prime}=M$. In addition, the scenario with a jamming signal present in the system provides motivation for the multiple constraint case [10], which is stated in the following manner:

$$
\min _{\mathbf{w}} \mathbf{w}^{H} \hat{\mathbf{R}}_{M} \mathbf{w} \quad \text { subject to } \quad \mathbf{w}^{H} \mathbf{A}=\mathbf{c} .
$$

If $\hat{\mathbf{R}}_{M}$ is an $(M \times M)$ Hermitian positive definite matrix and assuming $\mathbf{A}$ has full column rank equal to $\kappa$, where $\kappa \leq M$, then the unique solution to (13) is given by [11]

$$
\mathbf{w}_{C}=\hat{\mathbf{R}}_{M}^{-1} \mathbf{A}\left\{\mathbf{A}^{H} \hat{\mathbf{R}}_{M}^{-1} \mathbf{A}\right\}^{-1} \mathbf{c}^{H} .
$$

Three example LCMV beam patterns are presented in Fig. 2, with the gain shown in decibels. A 16-element ULA with $\lambda / 2$ element spacing was used in a system with 16 subscribers transmitting spread BPSK signals and an average SNR of $30 \mathrm{~dB}$ at each array element, based on an AWGN channel with zero mean and $\sigma^{2}$ variance so that

$$
\mathrm{SNR}=10 \log _{10} \frac{E_{b}}{\sigma^{2}}
$$

where $E_{b}$ is the energy per bit. Note that the ULA is aligned along an azimuth angle of $0^{\circ}$, resulting in the observed symmetry in the patterns. As designed, the method has resulted in unity gain toward the SOI. However, the LCMV method has also resulted in an adaptively adjusted beam pattern based on the received signal. Because the AWGN SNR is relatively high, the MAI generated by the other subscribers in the system has become the main source of interference. Thus, the result depicted in Fig. 2(a) has reduced sidelobes toward the group of subscribers located in the proximity of $150^{\circ}$ at the expense of increased noise gain elsewhere. This effect is also demonstrated in Fig. 2(b), where the same system was used with the exception that the average AWGN SNR is $5 \mathrm{~dB}$. As a result of increased AWGN, the LCMV method produces a beam pattern more similar to that of the Fourier method, where the main lobe has unity gain toward the SOI while the sidelobe gains are more evenly distributed. In this case, the MAI power has remained constant while the omnidirectional AWGN power has been increased, resulting in a beam pattern with reduced noise gain. In summary, the Fourier method results in the same beam pattern without regard to the received signal, while the LCMV method may change drastically to accommodate the environment. Further, as the SNR increases, the LCMV method results in increased noise gain, which is an undesirable effect of the LCMV beamformer. Another example of this phenomenon is depicted in Fig. 2(c), where the same 30-dB environment was used, with only four subscribers present. The subscriber at $70^{\circ}$ has $40 \mathrm{~dB}$ more power relative to the SOI. An extra constraint has been applied to null the jamming subscriber. Thus, the LCMV method attempts to mitigate the increased MAI of the jammer by generating a null toward the interfering signal. The noise gain, however, has increased significantly.

\section{B. Amplitude and Phase Estimation}

Motivated by the noise-gain issue present in the LCMV filter, the amplitude and phase-estimation (APES) filter was first introduced in Li et al. [12] and revisited in Stoica et al. [13] for applications to synthetic aperture radar (SAR). The APES filter was extended to the multiple look (i.e., spatial) scenario by Gini et al. [14] with regard to SAR interferometry, utilizing a similar derivation to that which follows.

Adapting to the spatial case, the signal is assumed to be a point source and constant over the period of a chip. Therefore, the array output, when the beam pattern is directed toward the SOI, should have a sinusoidal form. As a result, the method is derived by minimizing the error between the SOI during a single chip period and a sinusoidal model. Recall that no signal model was assumed with the LCMV method. As in the LCMV case, the error minimization is constrained by the requirement that 


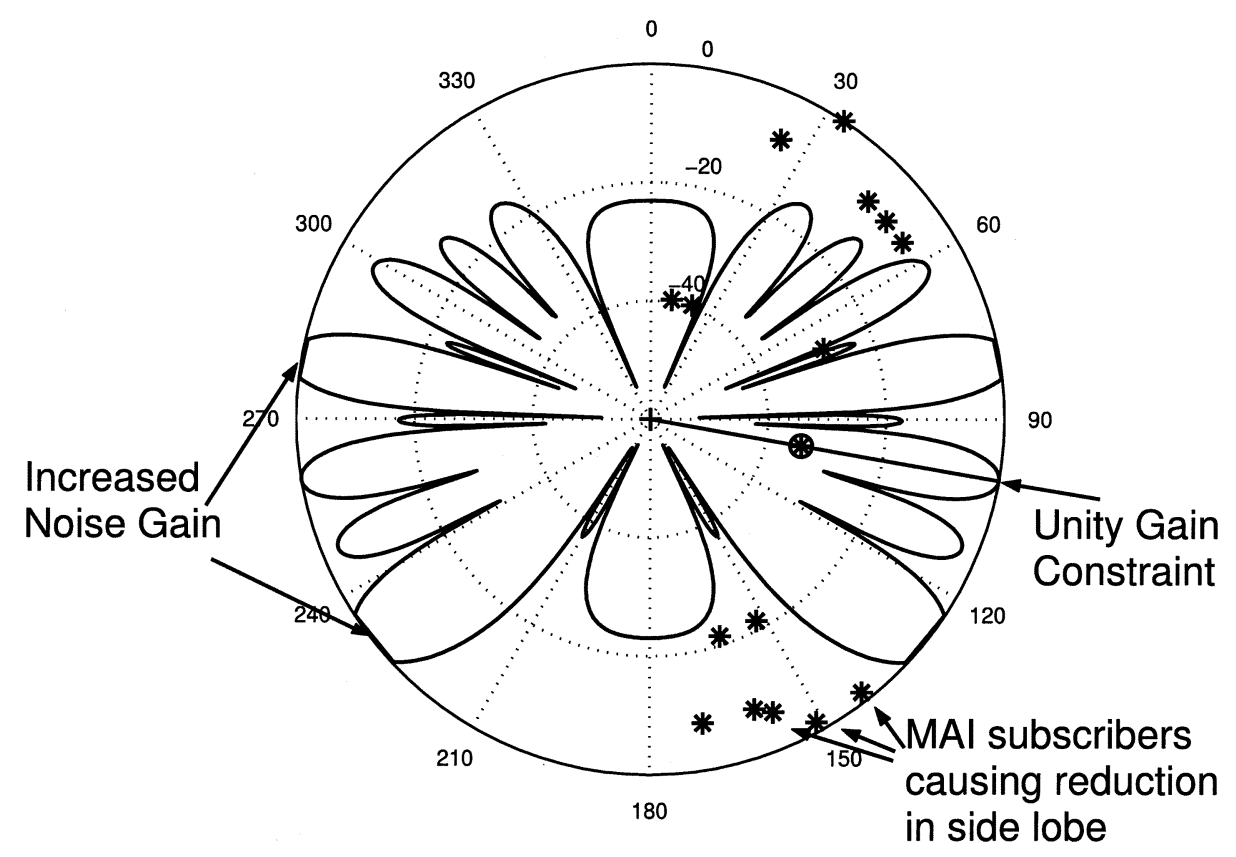

(a)

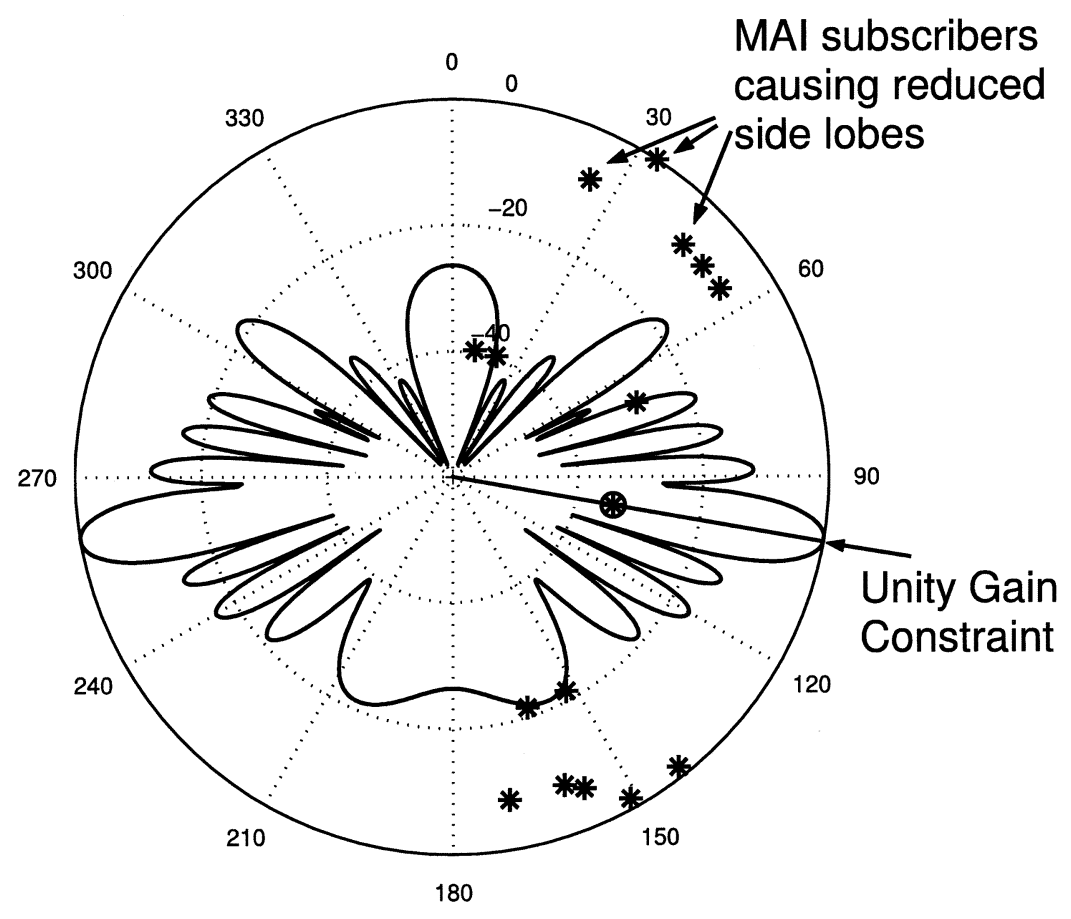

(b)

Fig. 2. Three examples of LCMV beam patterns. Note the difference between (a) the 30-dB environment and (b) the 5-dB environment. In example (c), interference from a jamming source may be mitigated with an extra constraint, depicted with an extra radial line, at the expense of increased noise gain elsewhere. (a) 30 -dB AWGN SNR. (b) 5-dB AWGN SNR.

there be unity gain at a particular DOA chosen to be toward the SOI

$\min _{\mathbf{w}, \alpha} \frac{1}{L} \sum_{l=0}^{L-1}\left|\mathbf{w}^{H} \mathbf{u}_{l, i}-\alpha a_{l}(\theta, \phi)\right|^{2}$ subject tow ${ }^{H} \mathbf{a}_{0}=1$.

For clarification, the received data vector $\mathbf{u}_{i}$ is partitioned into $L$ subarrays $\mathbf{u}_{l, i}$ and $a_{l}(\theta, \phi)$ is the first element of the $l$ th subarray steering vector.
To assist in the derivation, define

$$
\mathrm{g}_{i}(\theta, \phi)=\frac{1}{L} \sum_{l=0}^{L-1} \mathbf{u}_{l, i} a_{l}^{*}(\theta, \phi) .
$$

Then the minimization portion of the equation may be rewritten as

$$
\begin{aligned}
& \frac{1}{L} \sum_{l=0}^{L-1}\left|\mathbf{w}^{H} \mathbf{u}_{l, i}-\alpha a_{l}(\theta, \phi)\right|^{2} \\
& \quad=\frac{1}{L} \sum_{l=0}^{L-1} \mathbf{w}^{H} \mathbf{u}_{l, i} \mathbf{u}_{l, i}^{H} \mathbf{w}-\alpha^{*} \mathbf{w}^{H} \mathbf{g}_{i}(\theta, \phi)
\end{aligned}
$$




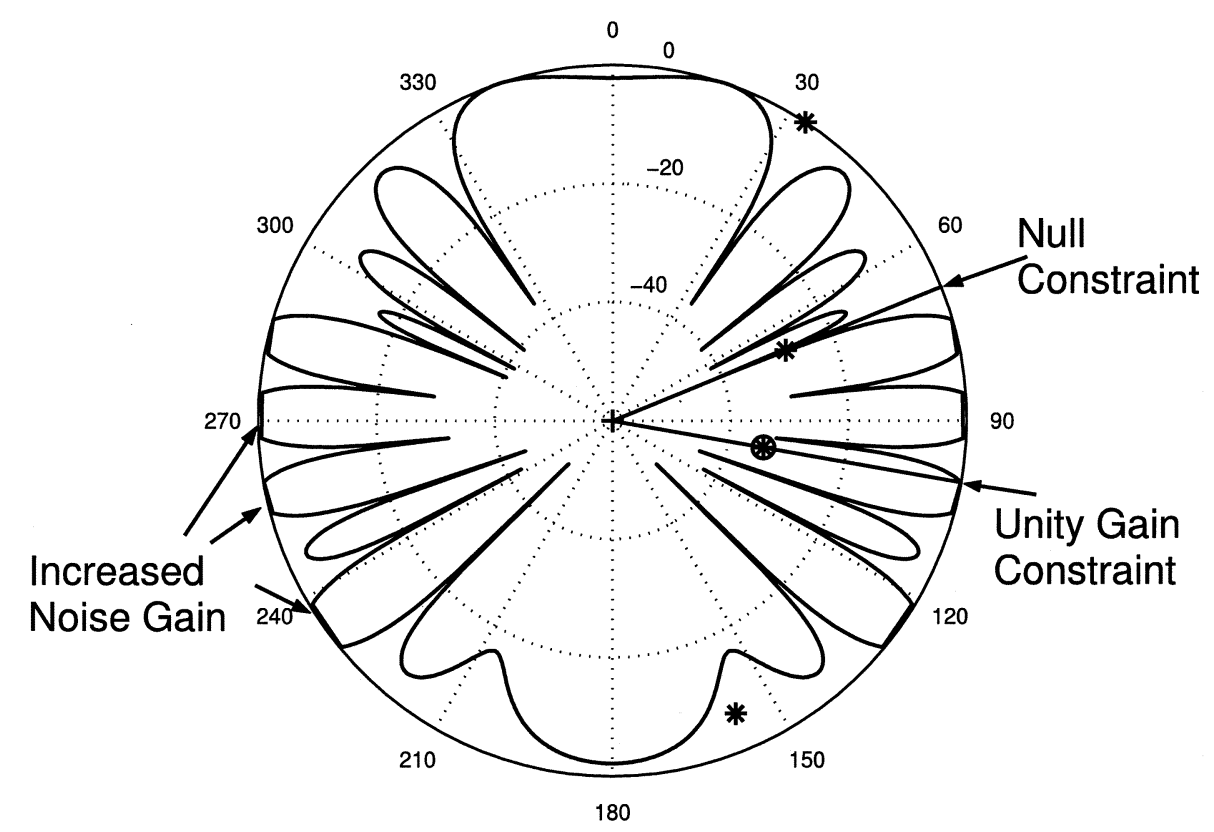

(c)

Fig. 2. (Continued.) Three examples of LCMV beam patterns. Note the difference between (a) the 30-dB environment and (b) the 5-dB environment. In example (c), interference from a jamming source may be mitigated with an extra constraint, depicted with an extra radial line, at the expense of increased noise gain elsewhere. (c) 30-dB AWGN SNR and 40-dB power increase from subscriber at $70^{\circ}$.

$$
\begin{aligned}
& -\alpha \mathbf{g}_{i}^{H}(\theta, \phi) \mathbf{w}+|\alpha|^{2} \\
= & \left|\alpha-\mathbf{w}^{H} \mathbf{g}_{i}(\theta, \phi)\right|^{2}+\frac{1}{L} \sum_{l=0}^{L-1} \mathbf{w}^{H} \mathbf{u}_{l, i} \mathbf{u}_{l, i}^{H} \mathbf{w} \\
& -\left|\mathbf{w}^{H} \mathbf{g}_{i}(\theta, \phi)\right|^{2} .
\end{aligned}
$$

The minimization with respect to $\alpha$ is given by $\alpha=$ $\mathbf{w}^{H} \mathbf{g}_{i}(\theta, \phi)$, which is used to obtain the following minimization problem for $\mathbf{w}$ :

$$
\begin{array}{r}
\min _{\mathbf{w}} \frac{1}{L} \sum_{l=0}^{L-1} \mathbf{w}^{H} \mathbf{u}_{l, i} \mathbf{u}_{l, i}^{H} \mathbf{w}-\left|\mathbf{w}^{H} \mathbf{g}_{i}(\theta, \phi)\right|^{2} \\
\text { subject to } \quad \mathbf{w}^{H} \mathbf{a}_{0}=1 .
\end{array}
$$

This new minimization argument may be written as

$$
\begin{aligned}
& \frac{1}{L} \sum_{l=0}^{L-1} \mathbf{w}^{H} \mathbf{u}_{l, i} \mathbf{u}_{l, i}^{H} \mathbf{w}-\left|\mathbf{w}^{H} \mathbf{g}_{i}(\theta, \phi)\right|^{2} \\
& \quad=\mathbf{w}^{H}\left(\frac{1}{L} \sum_{l=0}^{L-1} \mathbf{u}_{l, i} \mathbf{u}_{l, i}^{H}-\mathbf{g}_{i}(\theta, \phi) \mathbf{g}_{i}^{H}(\theta, \phi)\right) \mathbf{w} .
\end{aligned}
$$

Now, define the matrix $\mathbf{G}_{i}(\theta, \phi)=\mathbf{g}_{i}(\theta, \phi) \mathbf{g}_{i}^{H}(\theta, \phi)$, which results in

$$
\begin{aligned}
\mathbf{G}_{i}(\theta, \phi) & =\left(\frac{1}{L} \sum_{\tilde{l}=0}^{L-1} \mathbf{u}_{\tilde{l}, i} a_{\tilde{l}}^{*}(\theta, \phi)\right)\left(\frac{1}{L} \sum_{\hat{l}=0}^{L-1} \mathbf{u}_{\hat{l}, i}^{H} a_{\hat{l}}(\theta, \phi)\right) \\
& =\frac{1}{L^{2}} \sum_{\tilde{l}=0}^{L-1} \sum_{\hat{l}=0}^{L-1} \mathbf{u}_{\tilde{l}, i} \mathbf{u}_{\hat{l}, i}^{H} a_{\tilde{l}}^{*}(\theta, \phi) a_{\hat{l}}(\theta, \phi) .
\end{aligned}
$$

Next, define

$$
\hat{\mathbf{Q}}_{i}(\theta, \phi)=\frac{1}{L} \sum_{l=0}^{L-1} \mathbf{u}_{l, i} \mathbf{u}_{l, i}^{H}-\mathbf{G}_{i}(\theta, \phi)
$$

which is valid during the period of a single chip $i$. As noted previously (assuming ergodicity and using the temporal average equation)

$$
\begin{aligned}
\hat{\mathbf{Q}}(\theta, \phi) & =\frac{1}{N} \sum_{i=0}^{N-1} \hat{\mathbf{Q}}_{i}(\theta, \phi) \\
& =\hat{\mathbf{R}}_{M^{\prime}}-\mathbf{G}(\theta, \phi) .
\end{aligned}
$$

It can be shown that [see (24) and (25) at bottom of the page] where $\mathbf{S}(\theta, \phi)$ is the $\left(M^{\prime} \times M\right)$ matrix defined in (25).

$$
\begin{aligned}
& \mathbf{G}(\theta, \phi)=\frac{\mathbf{S}(\theta, \phi) \hat{\mathbf{R}}_{M} \mathbf{S}(\theta, \phi)^{H}}{L^{2}},
\end{aligned}
$$

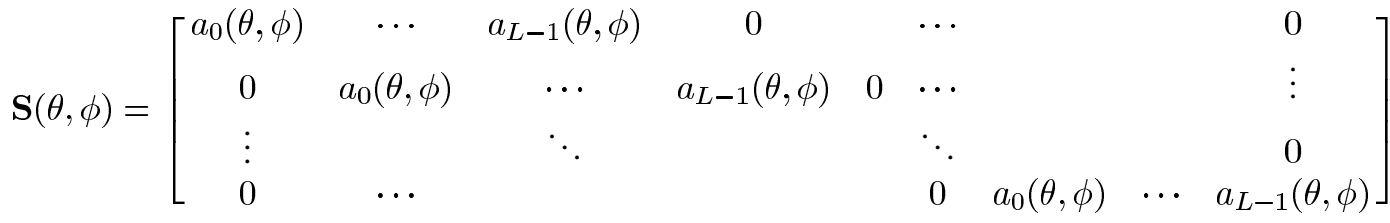




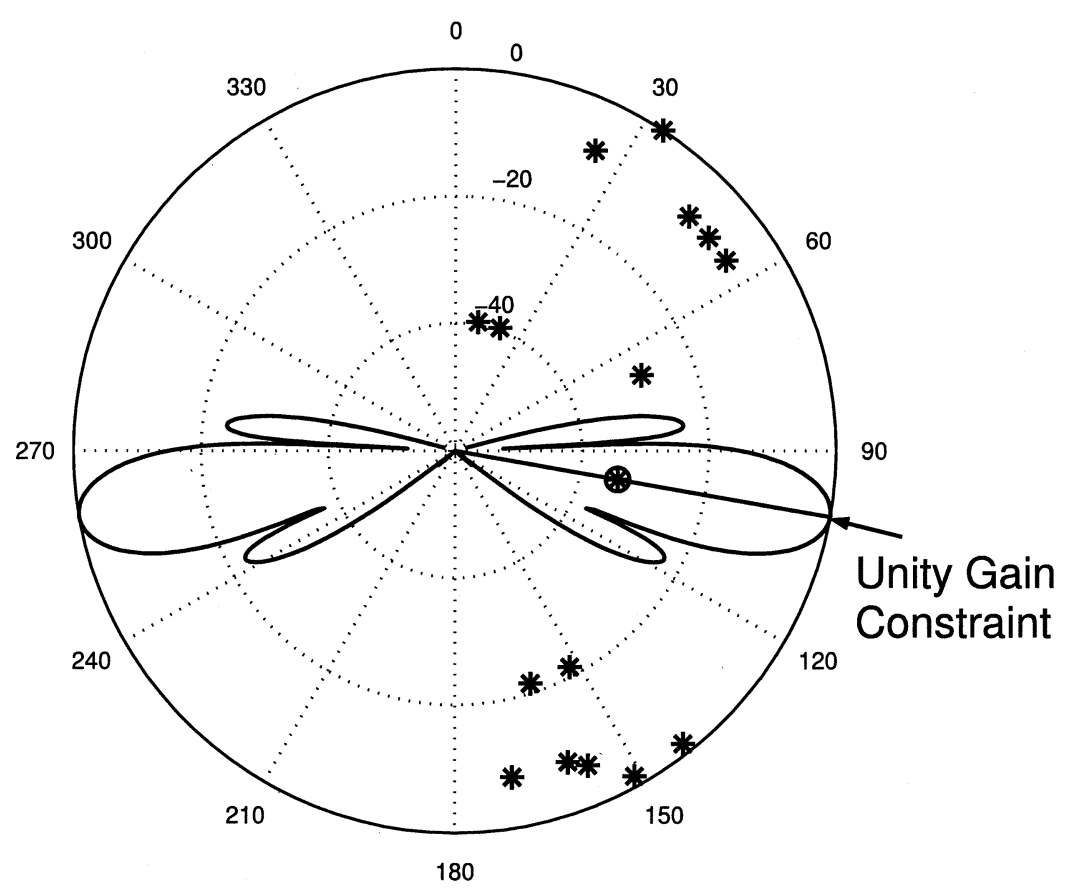

(a)

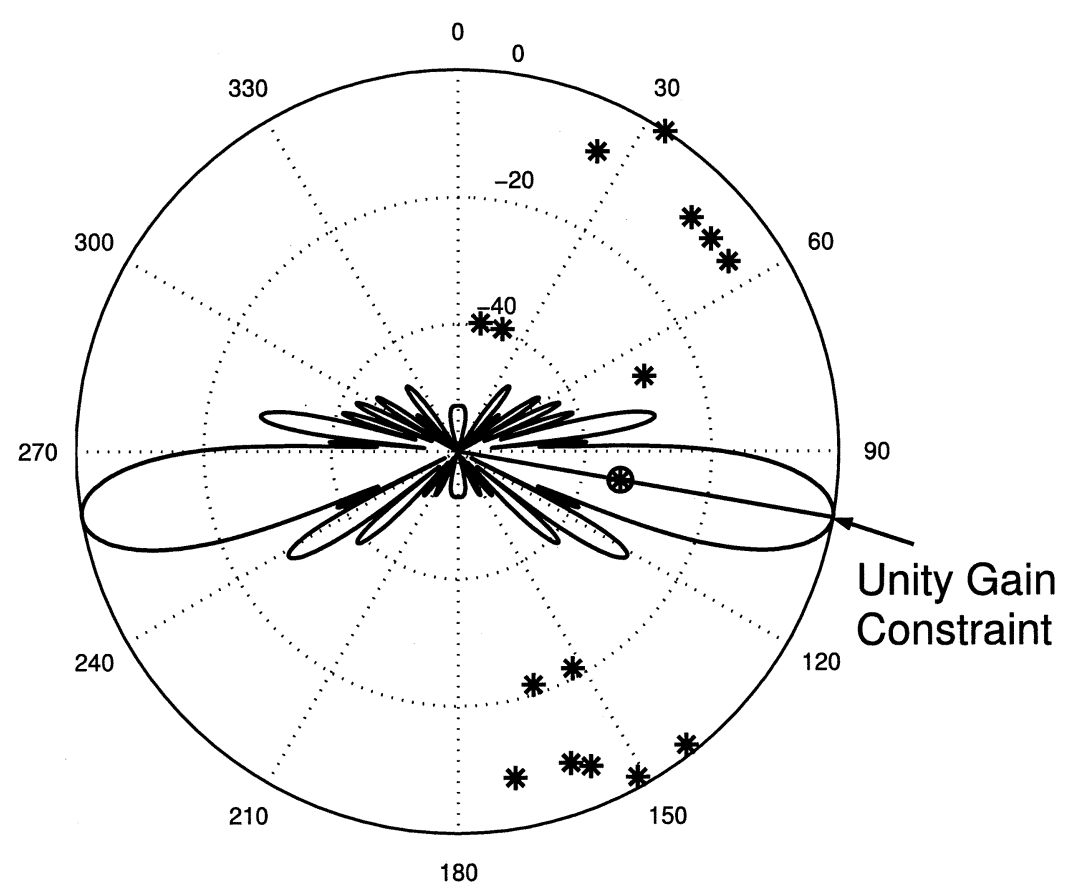

(b)

Fig. 3. Three examples of APES beam patterns generated using the same parameters as the LCMV beam patterns from Fig. 2. Notice that, compared to LCMV, the noise gain is considerably reduced at the expense of a reduction in spatial resolution. (a) 30-dB AWGN SNR. (b) 5-dB AWGN SNR.

For optimal performance, the APES filter length $M^{\prime}$ is usually chosen to be $M / 2$ [12]. Of course, angular resolution suffers for shorter filter lengths and, although not addressed in the present research, recent work has been performed to mitigate this degradation in resolution by combining the advantages of APES and the original LCMV methods [15]. Specifically, it was shown that there is a significant advantage to obtaining a DOA estimate from the LCMV method prior to applying the APES filter, due to a bias present in the APES DOA estimator.
The final problem statement has a similar form to the LCMV case and is given by

$$
\min _{\mathbf{w}} \mathbf{w}^{H} \hat{\mathbf{Q}}_{0} \mathbf{w} \quad \text { subject to } \quad \mathbf{w}^{H} \mathbf{a}_{0}=1
$$

where $\hat{\mathbf{Q}}_{0}$ is a simplified notation for $\hat{\mathbf{Q}}\left(\theta_{0}, \phi_{0}\right)$ and results in the APES beamformer

$$
\mathbf{w}_{A}=\hat{\mathbf{Q}}_{0}^{-1} \mathbf{a}_{0}\left\{\mathbf{a}_{0}^{H} \hat{\mathbf{Q}}_{0}^{-1} \mathbf{a}_{0}\right\}^{-1} .
$$




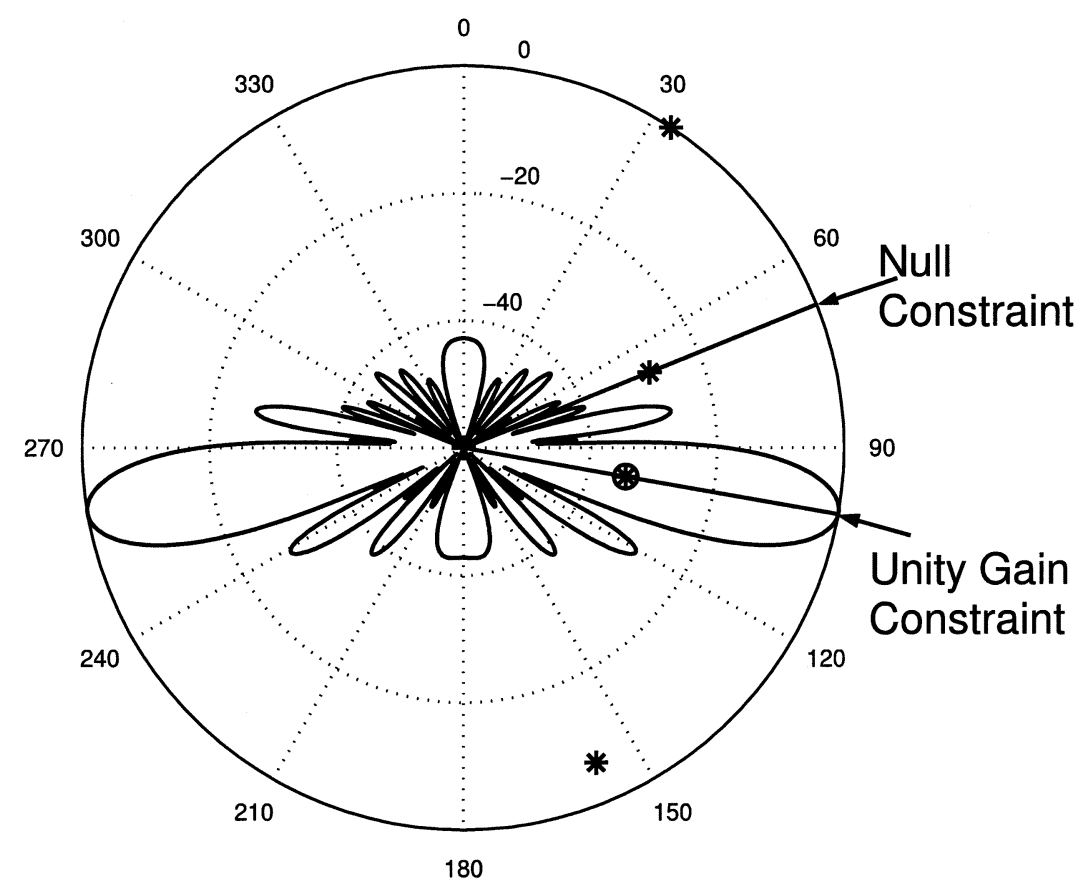

(c)

Fig. 3. (Continued.) Three examples of APES beam patterns generated using the same parameters as the LCMV beam patterns from Fig. 2. Notice that, compared to LCMV, the noise gain is considerably reduced at the expense of a reduction in spatial resolution. (c) 30-dB AWGN SNR and 40-dB power increase from subscriber at $70^{\circ}$.

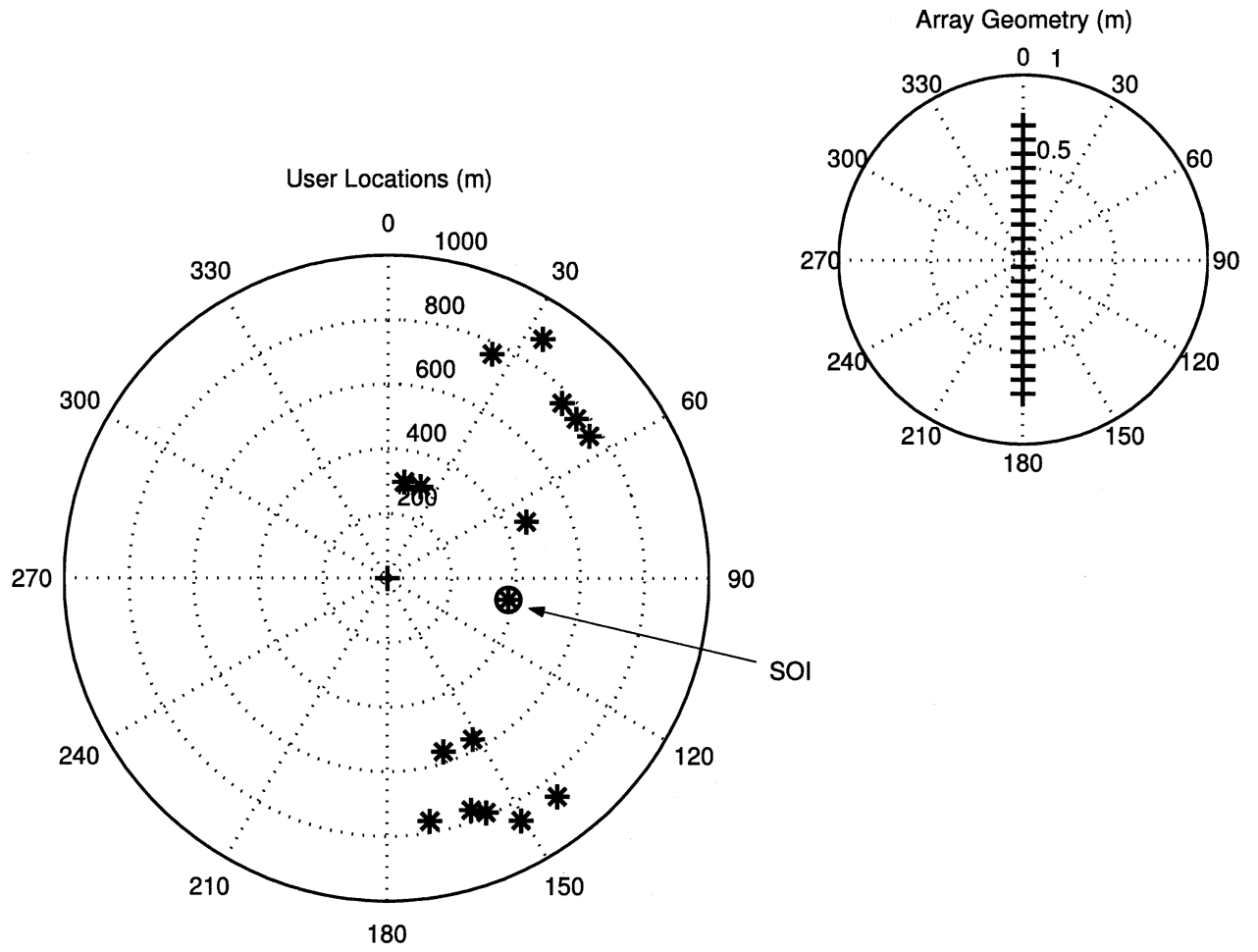

Fig. 4. Set of perfect power-control simulations use the same system geometry. In the user-locations plot, each of the 16 subscribers are identified by the symbol $*$, with the SOI further identified with the addition of a circle. Where appropriate, the receiver employs the 20 -element ULA with $\lambda / 2$ spacing, shown in the array geometry plot.

Note that $\mathbf{w}_{A}$ has length $M^{\prime}$. As a result, $\mathbf{w}_{A}$ is shifted through the $M$ signals that make up the data vector. Alternatively, by simple matrix manipulation it is possible to derive the composite weight vector of length $M$ resulting from the shifting process. 


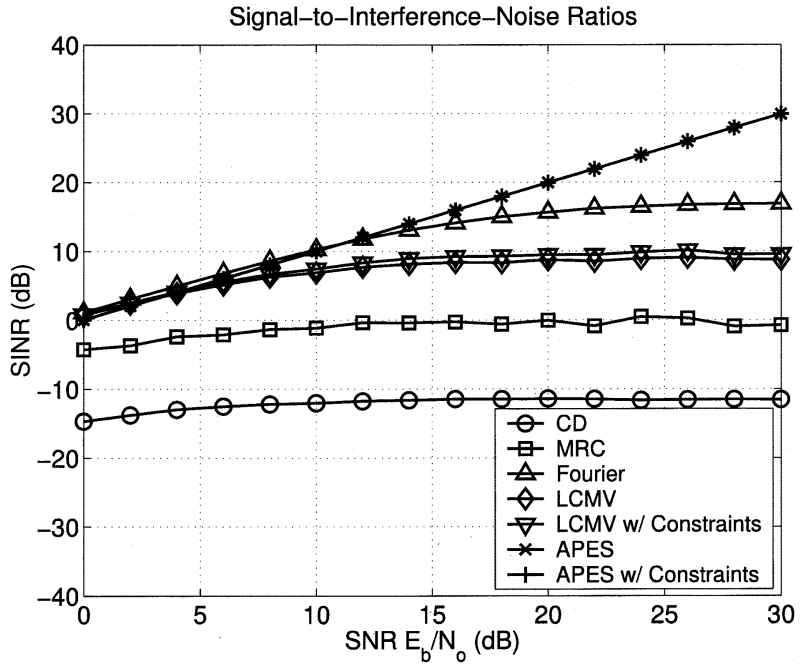

(a)

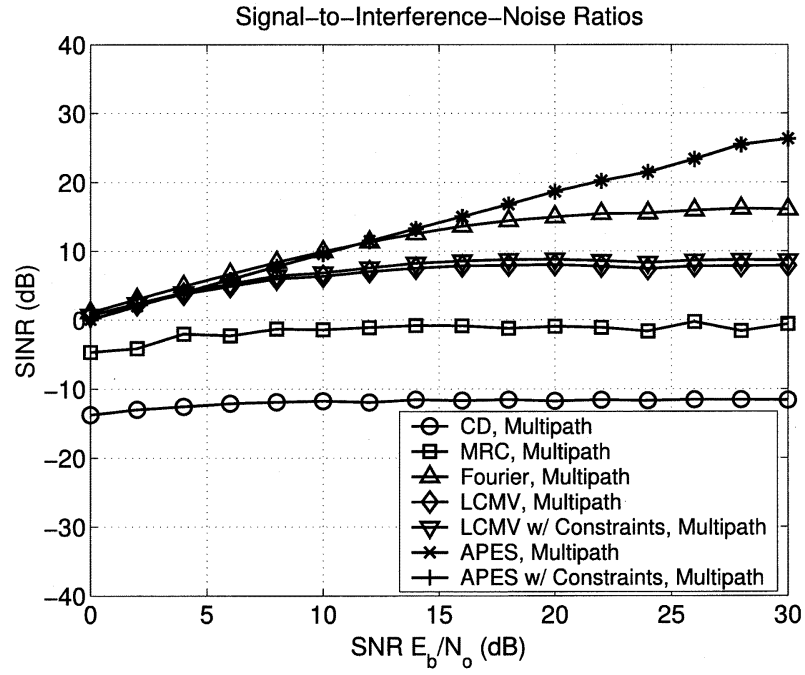

(b)

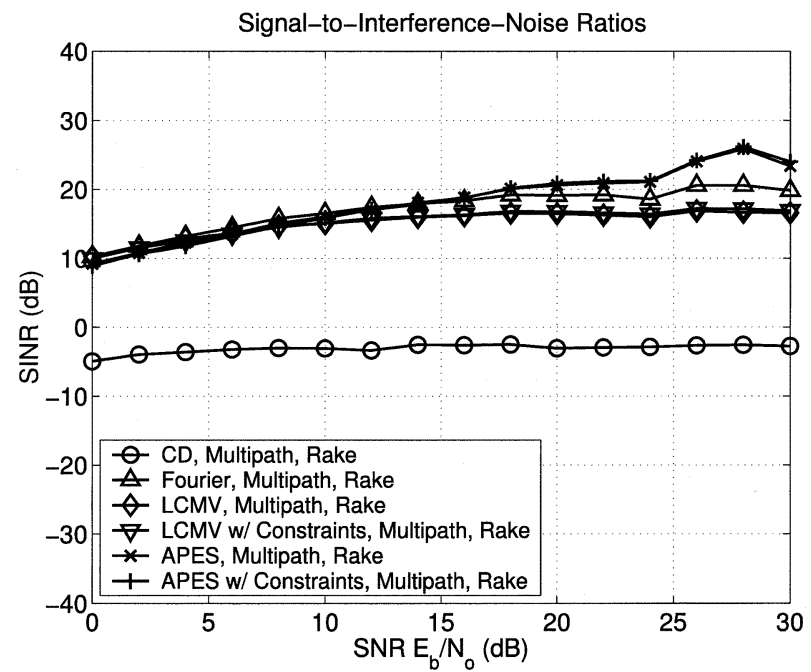

(c)

Fig. 5. Results of three sets of perfect power-control SINR simulations when the DOA of the subscribers is assumed to be known. Each set contains a CD, MRC, Fourier beamformer, LCMV beamformer with and without extra constraints, and APES beamformer with and without extra constraints. In (a), only AWGN is present in the channel and in (b) both AWGN and 15 multipath components are present in the channel. The simulation in (c) uses the same channel as that in (b) and changes the receiver to a three-finger RAKE. Note that there is no MRC RAKE receiver implementation. (a) AWGN, (b) AWGN and multipath, and (c) AWGN and multipath with three-finger RAKE.

As in the LCMV case, additional deterministic constraints can be applied to (26), resulting in the following constrained optimization problem:

$$
\min _{\mathbf{w}} \mathbf{w}^{H} \hat{\mathbf{Q}}_{0} \mathbf{w} \quad \text { subject to } \quad \mathbf{w}^{H} \mathbf{A}=\mathbf{c} .
$$

The solution for $\mathbf{w}$ for the multiple-constrained APES beamformer is given by

$$
\mathbf{w}_{A}=\hat{\mathbf{Q}}_{0}^{-1} \mathbf{A}\left\{\mathbf{A}^{H} \hat{\mathbf{Q}}_{0}^{-1} \mathbf{A}\right\}^{-1} \mathbf{c}^{H} .
$$

Similar to the LCMV presentation, three beam pattern examples are presented in Fig. 3. Each beam pattern was generated with the same simulation parameters as the respective LCMV beam patterns in Fig. 2. In all three instances, the noise gain has been reduced considerably, resulting in more-consistent beam patterns. However, the spatial resolution has also been reduced, resulting in a wider main lobe.

\section{NUMERICAL SimUlationS}

In this section, numerical simulations are used to study the possible advantages of the use of antenna arrays on a single-site CDMA BS receiver. All results were generated via Monte Carlo simulations of a CDMA uplink of a single cell using BPSK modulation. Each simulation is initiated by specifying the system geometry (i.e., the array geometry, carrier frequency, and subscriber locations and powers). During a single iteration (i.e., trial) of the simulation, a fixed number of source data bits are randomly generated for each user and are formed into a BPSK signal. These information bits are then combined with a subscriber pilot signal via length-two Walsh codes. Each subscribers' composite pilot/data signal is then spread with length-15 Gold codes [16]. Subsequently, all of the spread data sequences from the various users are randomly chip delayed (except for user zero, which is always assumed to be chip synchronized) and are mixed with an appropriate 

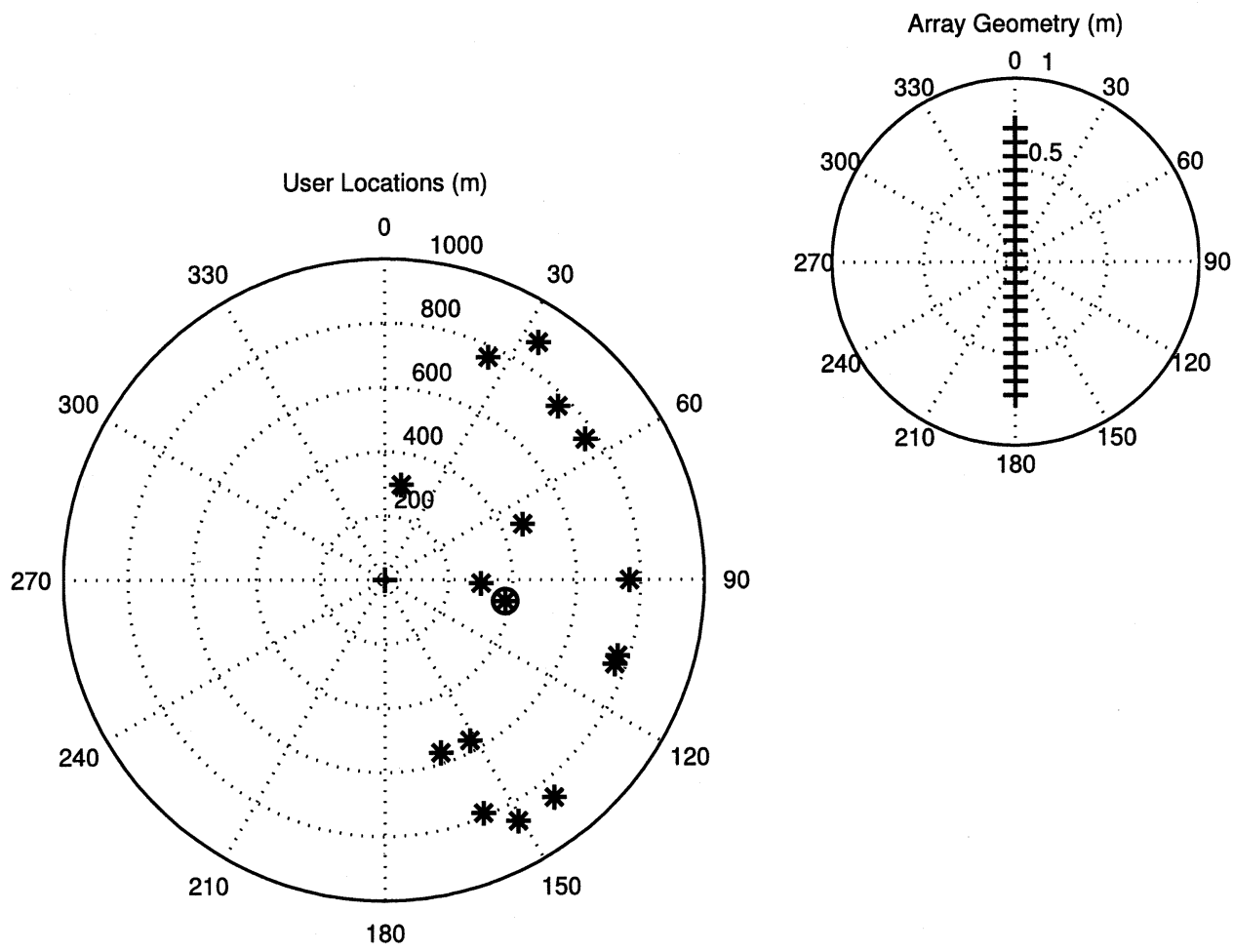

Fig. 6. To account for a les
remains a 20-element ULA.

steering vector based on the location of each user. Finally, the signals from all users are summed into a single data stream, which is passed through an AWGN channel with in-phase and quadrature noise components and may include a multipath environment using the geometrically based single-bounce elliptical model (GBSBEM) vector channel model [1].

The noise-corrupted chips are processed at the BS receiver array, which performs spatial sampling of the signal. Assuming known DOA of the SOI, the optimal array weights are then estimated. The array weights are used to form a beam toward the SOI, thus recovering the channelized data of interest. The remaining steps in the receiver perform dechannelization and remove the pilot signal. The recovered information signal is then used to estimate the SINR.

Each simulation is performed under equal conditions for the following detector types: matched filter conventional detector (CD), maximal ratio combiner (MRC), Fourier, LCMV, LCMV with two additional constraints, APES, and APES with two additional constraints. Note that the latter five detector types refer to the respective types of array beamforming. Unless explicitly stated, the following parameter settings are used throughout the simulations for each detector type:

1) pilot gain $=1.0$;

2) carrier frequency $=1960 \mathrm{MHz}$;

3) data bits transmitted per trial $=50$;

4) simulation-ending criterion $=100$ trials;

5) except for the $\mathrm{CD}$, the receiver employs a 20 -element ULA with $\lambda / 2$ spacing;

6) for both APES detectors, $M^{\prime}=M / 2$ (i.e., 10 elements). The simulation results presented containing a multipath environment that used the following GBSBEM parameter settings:
1) 15 multipath components with the line of sight (LOS) component present;

2) path-loss exponent $=2.0$;

3) reflection loss $=3.0 \mathrm{~dB}$;

4) excess chip delay $=15$ chips.

Note that, similar to how the AWGN process is sampled, the multipath components are regenerated for each trial.

\section{A. Perfect Power Control and Known Direction of Arrival}

The first simulation begins with the system geometry depicted in Fig. 4, which is the initial configuration used in all simulations with appropriate modifications applied where necessary. Shown in the figure are the subscriber locations relative to the antenna array, in addition to a close-up view of the array itself. Note that each subscriber is represented by the symbol $*$ and that each antenna element is represented by the symbol + . The simulation software operates on the basis that there is one SOI and that all other subscribers are considered interference. Thus, the SOI subscriber is highlighted in the user-locations plot with a circle. Because a ULA is used in the simulations, the subscriber locations have been restricted to one side of the array.

Fig. 5 contains three resulting sets of perfect power-control SINR simulations when the DOA of the subscribers is known precisely. Each plot contains the SINR results for a range of SNR, where SINR is defined as

$$
\mathrm{SINR}=\frac{E_{\mathrm{SOI}}}{E_{\mathrm{MAI}}+E_{\mathrm{ISI}}+\sigma^{2}}
$$

where $E_{\mathrm{SOI}}, E_{\mathrm{MAI}}$, and $E_{\mathrm{ISI}}$ are the energy values for the SOI, MAI, and ISI components, respectively. 


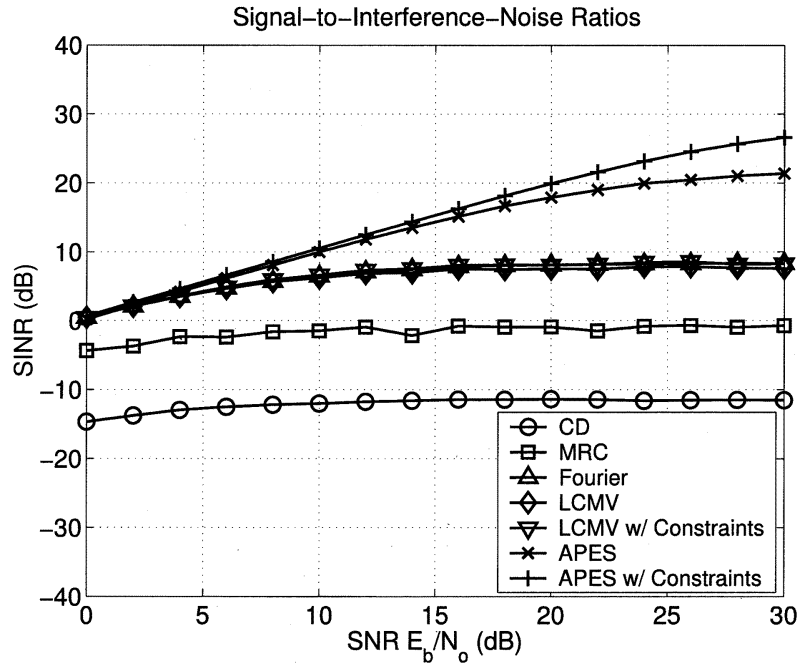

(a)

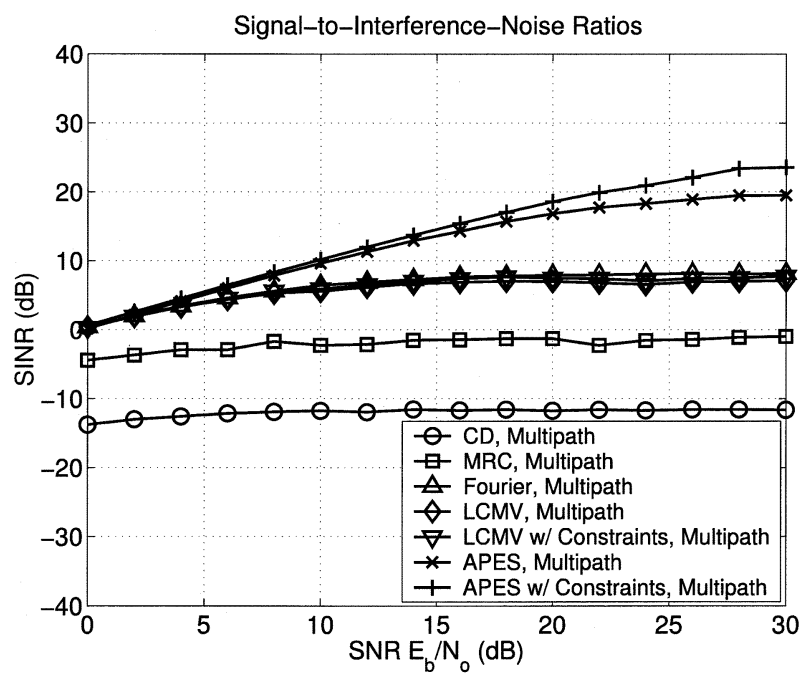

(b)

Fig. 7. For comparison with the results of Fig. 5(a) and (b), two sets of perfect power-control SINR simulations when the SOI is located in close proximity to interfering subscribers. All parameters are configured as in the simulations of Fig. 5(a) and (b), with the exception that four subscribers have been relocated. (a) AWGN. (b) AWGN and multipath.

Fig. 5(a) provides results for an AWGN-only channel. It should be noted that the CD generally had poor performance due to the length-15 Gold codes, which were used for channelization of the 16 subscribers. Even though the Gold codes are designed with relatively low cross-correlation, these codes are too short for practical use. However, because of their short length and asynchronous properties, they are attractive for use in these Monte Carlo simulations. Further, there is a $10-\mathrm{dB}$ increase in SINR when adding an array with MRC and an additional 10-dB increase when applying various beamforming methods. Among the beamforming methods, there are only marginal differences until the SNR rises above $10 \mathrm{~dB}$, at which point the Fourier and both APES methods increase beyond both LCMV methods. At the highest SNR of $30 \mathrm{~dB}$, the Fourier method increases SINR by $7 \mathrm{~dB}$ and both APES methods increase SINR by an additional $13 \mathrm{~dB}$.

Comparing Fig. 5(a) to (b), there is little difference among the performance of each detector type when enabling the multi-
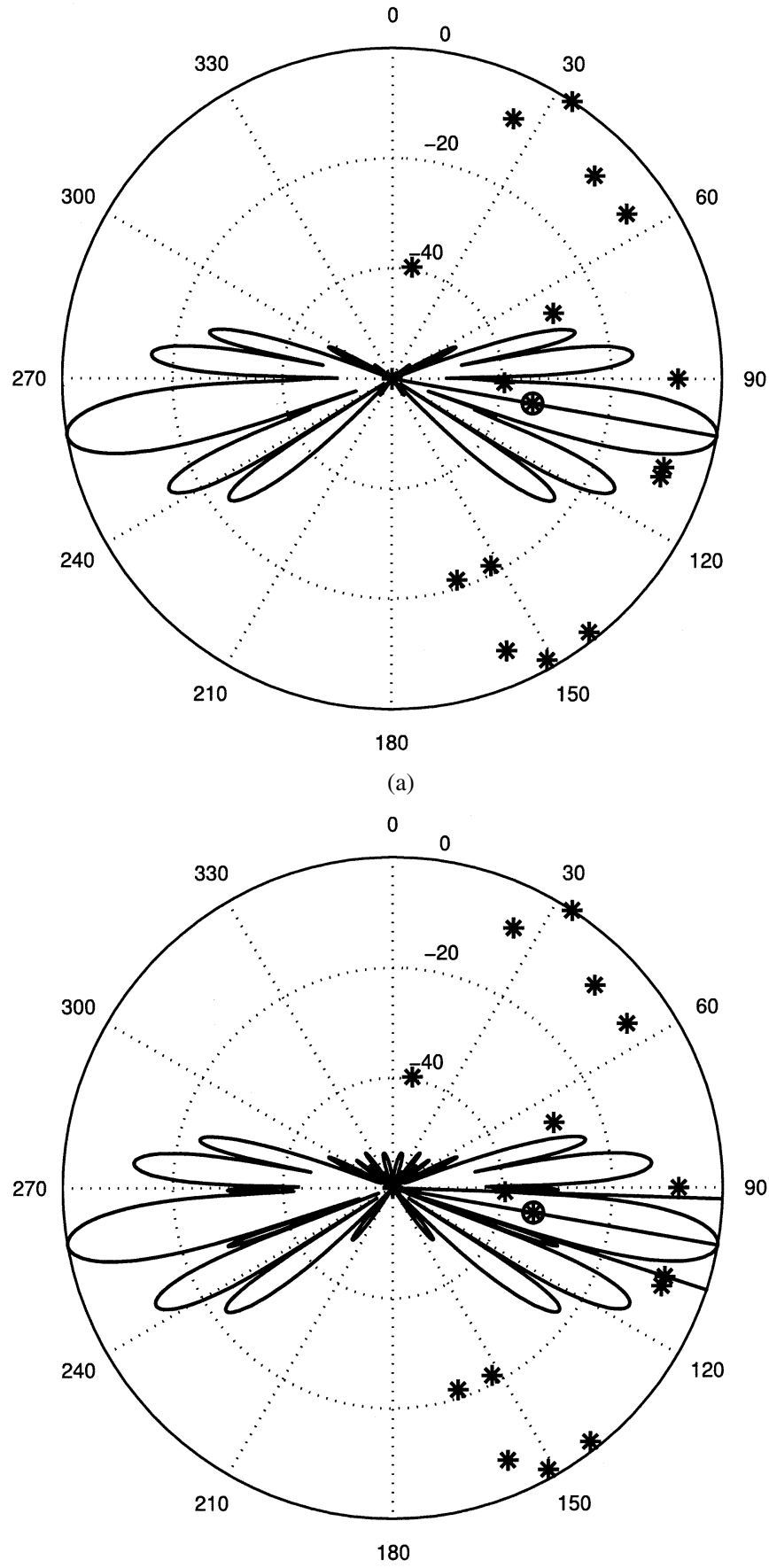

(b)

Fig. 8. Example beam patterns resulting from the two APES beamforming methods used in the perfect power-control simulation when the SOI is placed in close proximity to interfering subscribers. (a) APES, SNR $=30 \mathrm{~dB}$. (b) APES with two constraints, $\mathrm{SNR}=30 \mathrm{~dB}$.

path channel model. There is an overall slight decrease in SINR, which is expected. Finally, the scenario in (b) is simulated again in (c) with the addition of a three-finger RAKE receiver. The CD with the addition of a RAKE has an improvement of $9 \mathrm{~dB}$. While the array methods initially improve by $10 \mathrm{~dB}$, the LCMV methods have both improved the most at higher SNR while the APES methods have worsened slightly with the addition of the RAKE receiver. Both (b) and (c) scenarios include the LOS path, which contains the most significant portion of the signal. The 

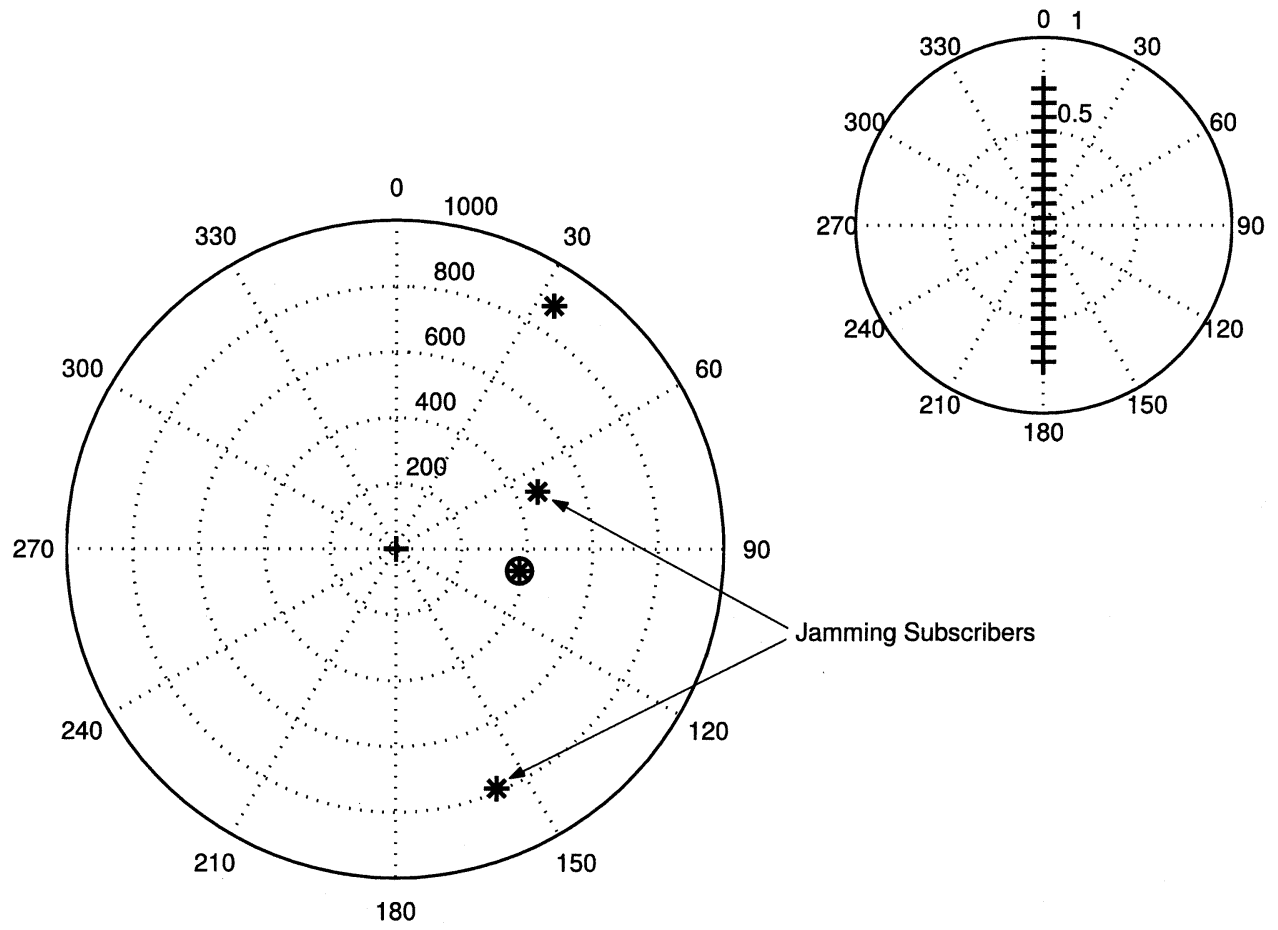

Fig. 9. System geometry for the jamming user simulations is generated from that of the perfect power-control simulations. The difference is that only the first four subscribers are present and that each subscriber on either side of the SOI is transmitting with 20-dB additional power.

latter simulation forms one RAKE finger precisely as that of the former simulation, in addition to two other RAKE fingers randomly distributed based on the GBSBEM model. While the two extra RAKE fingers include additional signal power, they also allow additional AWGN and possibly MAI into the RAKE combiner. At low SNR, the additional signal power is an obvious benefit, while at higher SNR the additional signal power is less evident versus the increase in noise power.

For the sake of validity, the work presented in Martone [17] depicts several results based in a length-15 Gold code environment and the research provided in Suard et al. [18] is based largely upon the concept of SINR. However, note that neither of these discussions directly relate to the simulations presented here.

\section{B. Perfect Power Control Within Close Proximity}

Because the subscriber positions depicted in Fig. 4 are used throughout the numerical simulations, an obvious question is the effect of the proximity of the other subscribers to the SOI. In an attempt to answer this question, a set of simulation results based on the system geometry depicted in Fig. 6 are presented in Fig. 7(a) and (b). As before, the data shown in (a) are the result of an AWGN-only channel and the data shown in (b) are the result of the additional multipath channel. Compared to the simulation results from Fig. 5(a) and (b), the CD and MRC detector types have nearly the same respective performances, demonstrating the lack of dependence upon the subscriber location. Of the array beamforming methods, all detector types result in the same respective SINR performance at low SNR, while at 30-dB SNR both LCMV simulations demonstrated the least amount of performance loss with $1 \mathrm{~dB}$. The Fourier beamforming method is shown to be the most affected by the close proximity of the interfering subscribers, as it has lost 10-dB SINR and is now performing approximately the same as the LCMV beamforming methods. The APES beamforming methods still provide the highest SINR; however, there is now a noticeable difference between the APES beamformer and the APES-with-two-extra-constraints beamformer. The former method, an example of which is depicted in Fig. 8(a), has a 9-dB decrease in performance while the latter method, shown in Fig. 8(b), only has a 3-dB decrease in performance. The overall loss in performance of the APES beamforming methods, as compared to the LCMV beamforming methods, may be attributed to the loss in angular resolution as a result of the APES filter length being $M / 2$. Thus, the main lobe in Fig. 8(a) and (b) is wider than the respective main lobes from a Fourier beam pattern or an LCMV beam pattern. When the additional constraints are applied, as in Fig. 8(b), the main lobe is narrower to accommodate the nearby nulls, resulting in an overall 6-dB performance gain. Also note that the sidelobes in Fig. 8(b) have a gain increase associated with them, as a result of the additional constraints.

The primary result from these simulations is the lack of performance degradation associated with the LCMV beamforming methods. Comparing the results from Fig. 5(a) and (b) to Fig. 7(a) and (b), the LCMV adaptive array detectors have demonstrated the least amount of dependence upon the subscriber locations.

\section{Jamming Subscribers and Known Direction of Arrival}

The next set of simulations modifies the original system geometry from Fig. 4 to that of Fig. 9, in which only the SOI and three other subscribers are active. In addition, the two subscribers adjacent to the SOI are transmitting with 20 -dB addi- 


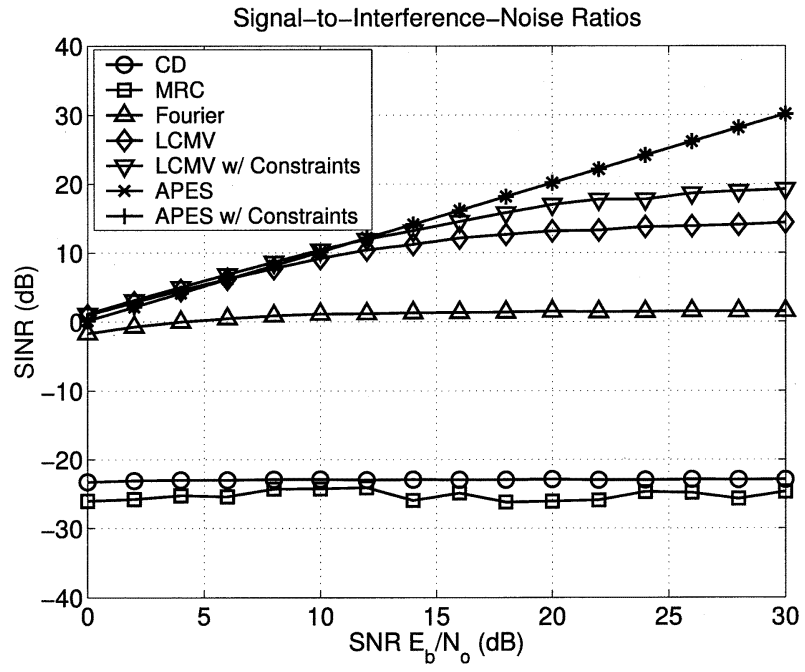

(a)

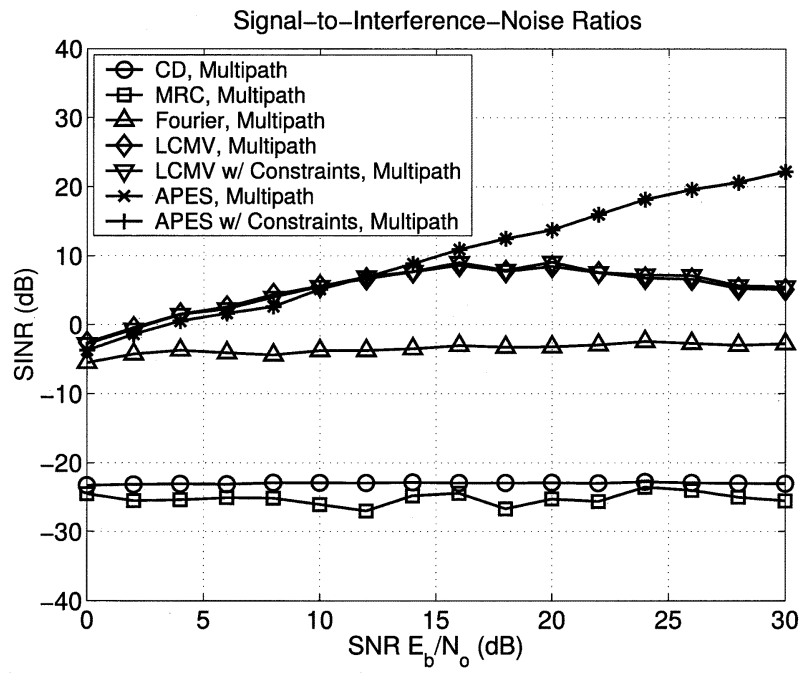

(b)

Fig. 10. Two sets of jamming user SINR simulations when the DOA of the subscribers is assumed to be known. Each set contains a CD, MRC, Fourier beamformer, LCMV beamformer with and without extra constraints, and APES beamformer with and without extra constraints. In (a), only AWGN is present in the channel and in (b) both AWGN and 15 multipath components are present in the channel. (a) AWGN. (b) AWGN and multipath.

tional power, thereby acting as jamming subscribers with respect to the SOI.

With many similarities between the simulations for Fig. 5(a) and (b) and Fig. 10(a) and (b), it is appropriate to compare the results to see how the jamming subscribers have affected the performance of the various algorithms. Note that there has been a reduction in the number of subscribers from the former to the latter simulations, the reason being that the jamming subscriber simulation was originally designed to compare the results between the LCMV and APES methods with the multiple constraint versions of the same methods.

Beginning with the $\mathrm{CD}$, the performance from the perfect power-control simulation to the jamming subscriber simulation has worsened by $10 \mathrm{~dB}$ at high SNR. Although the number of subscribers is fewer, the increased power has adversely affected the performance of the Gold codes. Even worse is the MRC, which has decreased performance by $27 \mathrm{~dB}$ at high SNR. Re- garding the beamforming techniques, both APES methods result in nearly the same performance for both simulation types, while Fourier has decreased by $16 \mathrm{~dB}$ due to the nonadaptive nature of the algorithm. Finally, both LCMV methods have performed better in the jamming subscribers case by as much as $10 \mathrm{~dB}$ for LCMV with two extra constraints. Further, it is evident from the jamming subscribers simulation that multiple constraints may be able to improve system performance.

\section{Number of Subscribers}

It is of interest to analyze how parameters other than SNR affect receiver performances. This simulation uses the same system geometry depicted in Fig. 4. However, the AWGN SNR is fixed at $20 \mathrm{~dB}$ and the total number of subscribers is allowed to vary. Specifically, the first data point results from the system operating with only the SOI subscriber transmitting (i.e., zero MAI). At each successive data point, another subscriber from the system geometry begins transmitting. Thus, as the number of subscribers transmitting increases, the overall system performance should worsen.

The simulation results are presented in Fig. 11, where (a) is the result of only AWGN and (b) contains multipath. In general, the results are as expected. However, it is interesting to note that the Fourier and both APES beamforming methods are generally unaffected by the increase in MAI, while both LCMV methods tend to worsen slightly more as subscribers are added to the system. It should be noted that these results represent only one fixed system geometry.

In the literature, the number of subscribers is often estimated since it can be used as a proxy for system capacity. Unfortunately, there is no common system type used for these studies. Further, there are many system variables that may be altered, leading to difficult comparisons. However, the general result is obvious in that as subscribers are added to the system, performance worsens [5], [19]-[24]. Thus, it is desired to decrease the amount by which the system performance degrades in relation to the number of active subscribers.

\section{E. Number of Elements}

Another parameter that may affect receiver performance is the number of elements present in the array. The simulation software collects data points for this simulation type by retrieving the array from persistent memory and storing a subsection of that array, beginning with the first element by itself. At each successive data point, the next element of the array is appended to the antenna before the set of trials are executed. While the original system geometry would perform adequately for this simulation, a 32-element ULA is used instead to allow for an extended view of the performance change due to number of elements. Note that the number of subscribers and their locations are the same as those from Fig. 4.

The results of the simulation are presented in Fig. 12, with the AWGN-only system in (a) and the multipath-enabled channel in (b). As was the case of the previous simulation type, the AWGN SNR is fixed at $20 \mathrm{~dB}$. Because this simulation requires changing the number of elements, there is no application to the $\mathrm{CD}$; thus, it is removed from consideration. Actually, each receiver type begins with a single element that should reduce to 


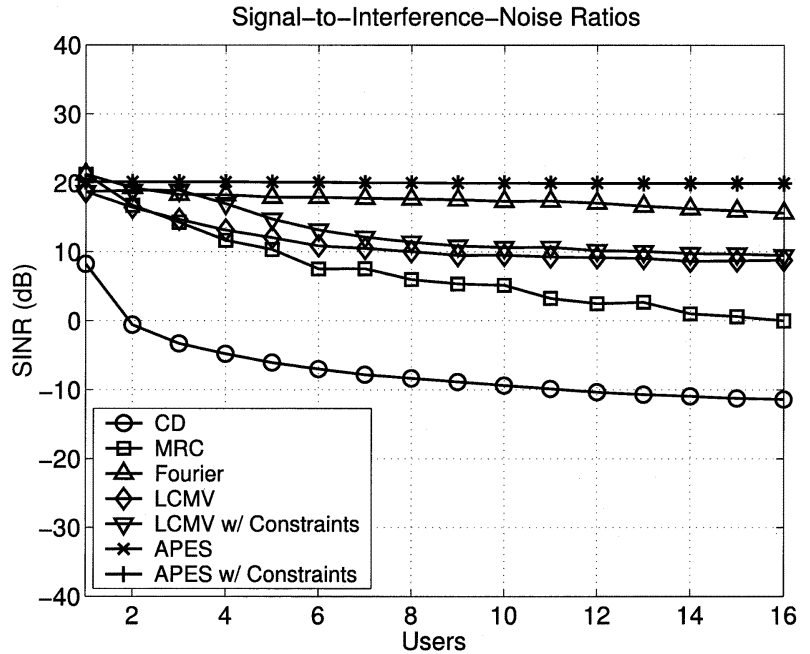

(a)

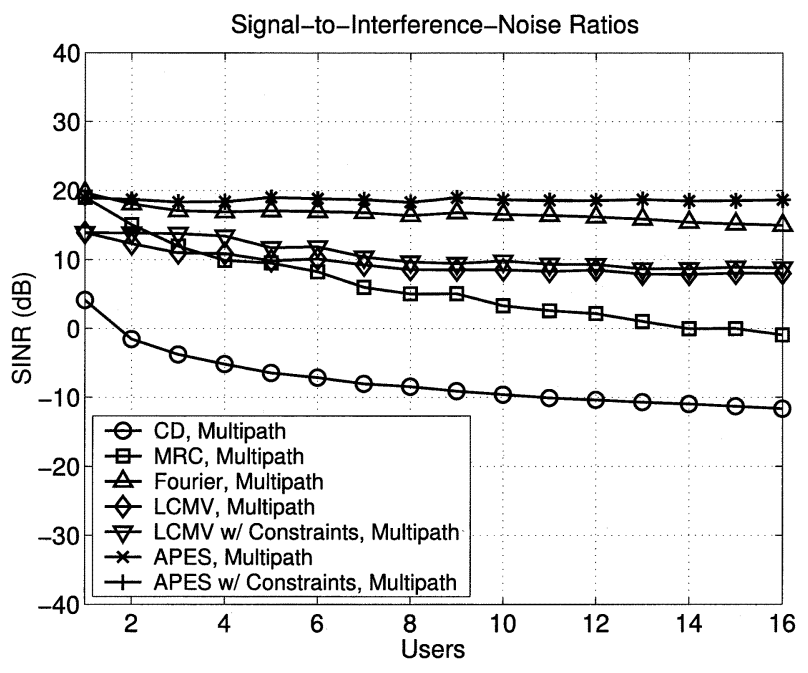

(b)

Fig. 11. Resulting SINR simulations when the subscribers from the system geometry of Fig. 4 are systematically increased. Again, (a) is the result of an AWGN-only channel and (b) is the result of an AWGN-plus-15 multipath component channel. (a) AWGN. (b) AWGN and multipath.

the CD. As can be seen from the results, every algorithm results in the same data point when there is only one element present in the array.

Intuitively, one would expect the receiver performance to improve with the addition of multiple elements. This result is demonstrated by the most general case of the MRC, where there is an initial rapid improvement of $8 \mathrm{~dB}$ from one to three elements, followed by a general improvement by $4 \mathrm{~dB}$ from three to 32 elements. Similarly, both APES algorithms follow the same smooth increase once there are six elements present in the array. Prior to that, the APES with two extra constraints method performs unreliably due to a lack of array resources. Both APES algorithms outperform the MRC method by $20 \mathrm{~dB}$, once there are six or more elements. The Fourier beamforming method results in a curve that is slightly below the APES results. However, the Fourier algorithm has a slight oscillation in the results due to the distribution of sidelobes as extra elements are added. As a new element is added to the array, a

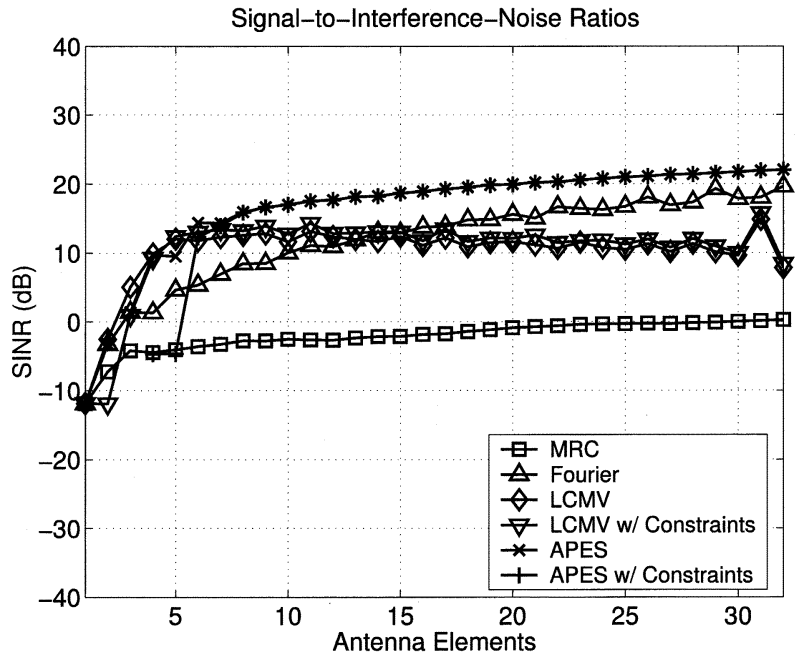

(a)

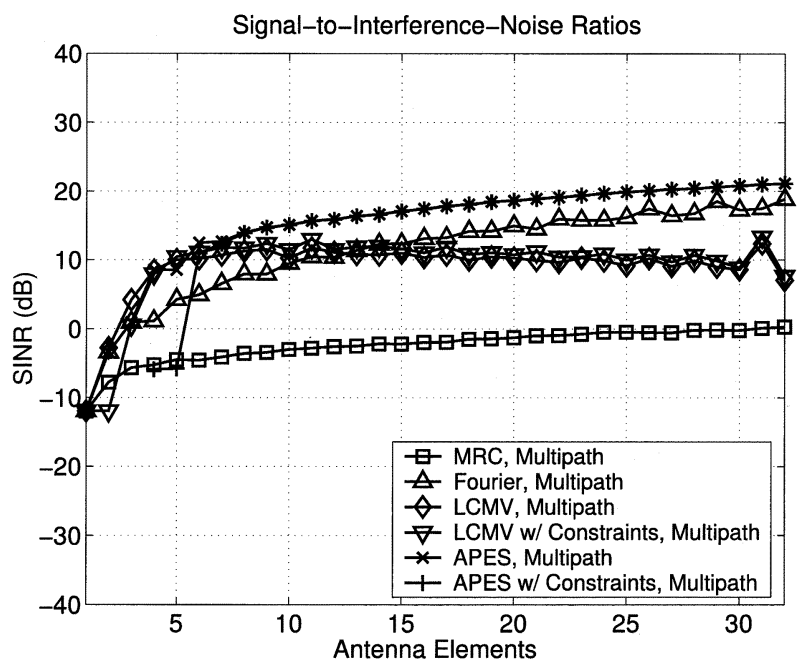

(b)

Fig. 12. Resulting SINR simulations when the number of antenna elements are added one at a time. For apparent reasons, the CD is excluded from these simulations. The results in (a) are the result of an AWGN-only channel and in (b) are the result of an AWGN-plus-15 multipath component channel. (a) AWGN. (b) AWGN and multipath.

sidelobe pointing at an interfering subscriber may appear, resulting in diminished performance. The most interesting results appear in the LCMV receiver structures. Up to 11 elements, the LCMV methods seem to follow the APES results, at which point there is a gradual performance decrease as elements are added. There also seems to be a slight oscillation in the data, similar to the Fourier results. In an attempt to explain this phenomenon, two beam patterns are presented in Fig. 13. The pattern in (a) is taken with ten elements present in the array and the pattern in (b) is the result of an array with 32 elements. Both beam patterns have unity gain toward the SOI and have attempted to attenuate the interfering subscribers. Obviously, the pattern in (a) has fewer sidelobes due to the reduced number of elements. Likewise, the pattern in (b) has many more sidelobes, many of which have higher gain associated with them. As has been the case in previous simulations, the LCMV method seems to achieve a certain limit. As further improvement to the system is applied, the results begin to worsen. Clearly, there is some volatility present 


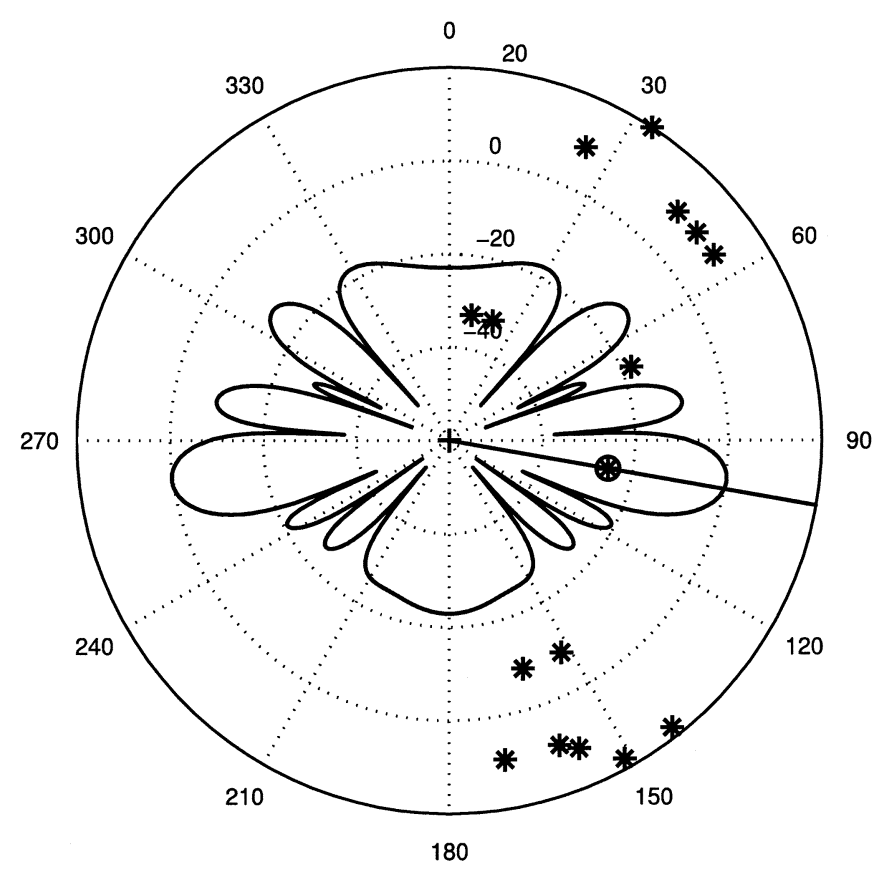

(a)

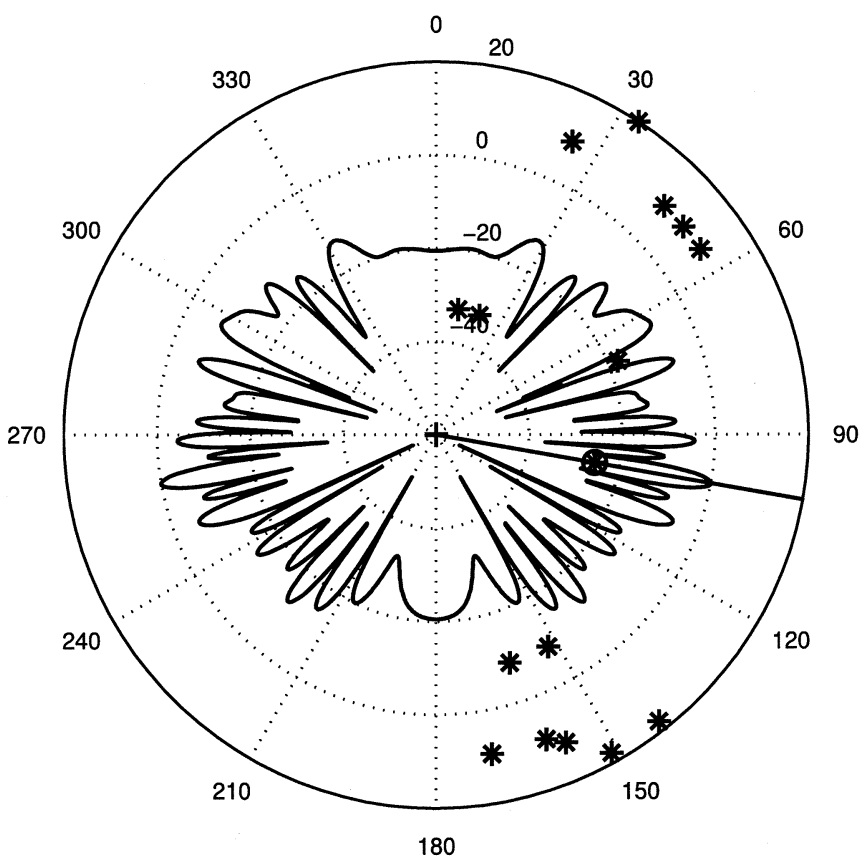

(b)

Fig. 13. Two LCMV beam patterns applied to the same system data. The pattern in (a) is the result of an array with ten elements and the pattern in (b) is the result of an array with 32 elements. In general, there is an increase in the number and magnitude of sidelobes with the second pattern. (a) Ten-element array. (b) 32-element array.

in the algorithm, which may depend on the specific geometry of this simulation.

This particular simulation is specific to antenna array research, as opposed to multiuser detector (MUD)-specific improvement methods. As with previous simulations, there is no single environment that is universal among the surveyed works; however, there are several results available that corroborate the results obtained in this section [6], [25], [26].

\section{CONCLUSION}

The primary goal of this work was to introduce and compare viable adaptive array methods for the mitigation of various forms of CDMA reverse channel interference [1], [27]. The APES temporal filter [12], [13] was adapted to the spatial case and was shown to have noise-gain advantages over more well-known methods [9]. Results from extensive numerical simulations were presented in an attempt to study the effects of important physical parameters within the communication system and channel. In general, the spatial APES beamformer demonstrated superior SINR performance with respect to several other receiver types under a variety of channel and system conditions. It is essential for modern communication systems to allow increased capacity while maintaining reasonable cost. Therefore, adaptive antenna array technology will play a pivotal role in future CDMA communication systems. It is the intent of this work to assist in considerations for future implementation challenges.

\section{REFERENCES}

[1] J. C. Liberti Jr. and T. S. Rappaport, Smart Antennas for Wireless Communications: IS-95 and Third Generation CDMA Applications. Englewood Cliffs, NJ: Prentice-Hall, 1999.

[2] S. Verdu, "Minimum probability of error for asynchronous Gaussian multiple-access channels," IEEE Trans. Inform. Theory, vol. IT-32, pp. 85-96, Jan. 1986.

[3] R. Lupas and S. Verdu, "Linear multiuser detectors for synchronous code-division multiple-access channels," IEEE Trans. Inform. Theory, vol. 35, pp. 123-136, Jan. 1989.

[4] S. Moshavi, "Multi-user detection for DS-CDMA communications," IEEE Commun. Mag., vol. 34, pp. 124-136, Oct. 1996.

[5] L. Fang and L. B. Milstein, "Successive interference cancellation in multicarrier DS/CDMA," IEEE Trans. Commun., vol. 48, pp. 1530-1540, Sept. 2000

[6] J. S. Thompson, P. M. Grant, and B. Mulgrew, "Smart antenna arrays for CDMA systems," IEEE Pers. Commun., vol. 3, pp. 16-25, Oct. 1996.

[7] M. Chryssomallis, "Smart antennas," IEEE Antennas Propagat. Mag., vol. 42, pp. 129-136, June 2000.

[8] B. D. Van Veen and K. M. Buckley, "Beamforming: a versatile approach to spatial filtering," IEEE ASSP Mag., pp. 4-24, Apr. 1988.

[9] J. Capon, "High-resolution frequency-wavenumber spectrum analysis," Proc. IEEE, vol. 57, pp. 1408-1418, Aug. 1969.

[10] O. L. Frost, "An algorithm for linearly constrained adaptive array processing," Proc. IEEE, vol. 60, pp. 926-935, Aug. 1972.

[11] P. Stoica and R. Moses, Introduction to Spectral Analysis. Englewood Cliffs, NJ: Prentice-Hall, 1997.

[12] J. Li and P. Stoica, "An adaptive filtering approach to spectral estimation and SAR imaging," IEEE Trans. Signal Processing, vol. 44, pp. 1469-1484, June 1996.

[13] P. Stoica, H. Li, and J. Li, "A new derivation of the APES filter," IEEE Signal Processing Lett., vol. 6, pp. 205-206, Aug. 1999.

[14] F. Gini and F. Lombardini, "Multilook APES for multibaseline SAR interferometry," IEEE Trans. Signal Processing, vol. 50, pp. 1800-1803, July 2002.

[15] A. Jakobsson and P. Stoica, "Combining Capon and APES for estimation of spectral lines," Circuits, Syst., Signal Processing, vol. 19, no. 2, pp. $159-169,2000$.

[16] J. S. Lee and L. E. Miller, CDMA Systems Engineering Handbook. Norwood, MA: Artech House, 1998.

[17] M. Martone, "Blind adaptive detection of DS/CDMA signals on timevarying multipath channels with antenna arrays using high-order statistics," IEEE Trans. Commun., vol. 48, pp. 1590-1600, Sept. 2000.

[18] B. Suard, A. F. Naguib, G. Xu, and A. Paulraj, "Performance of CDMA mobile communication systems using antenna arrays," in Proc. Int. Conf. Acoustics, Speech, and Signal Processing (ICASSP) '93, vol. IV, Apr. 1993, pp. 153-156.

[19] A. F. Naguib, A. Paulraj, and T. Kailath, "Capacity improvement with base-station antenna arrays in cellular CDMA," IEEE Trans. Veh. Technol., vol. 43, pp. 691-698, Aug. 1994. 
[20] H. Liu and M. D. Zoltowski, "Blind equalization in antenna array CDMA systems," IEEE Trans. Signal Processing, vol. 45, pp. 161-172, Jan. 1997.

[21] C. Z. W. H. Sweatman, B. Mulgrew, J. S. Thompson, and P. M. Grant, "Multiuser detection for CDMA antenna array receivers using spatial equivalence classes," Inst. Elect. Eng. Proc. Commun., vol. 147, no. 2, pp. 105-113, 2000.

[22] A. J. Paulraj and C. B. Papadias, "Space-time processing for wireless communications," IEEE Signal Processing Mag., vol. 14, pp. 49-83, Nov. 1997.

[23] Z. Rong, P. Petrus, T. S. Rappaport, and J. H. Reed, "Despread-respread multi-target constant modulus array for CDMA systems," IEEE Commun. Lett., vol. 1, pp. 114-116, July 1997.

[24] J. C. Liberti Jr. and T. S. Rappaport, "Analytical results for capacity improvements in CDMA," IEEE Trans. Veh. Technol., vol. 43, pp. 680-690, Aug. 1994.

[25] J. Choi, "A receiver of simple structure for antenna array CDMA systems," IEEE Trans. Veh. Technol., vol. 48, pp. 1332-1340, Sept. 1999.

[26] J. H. Winters, "Smart antennas for wireless systems," IEEE Pers. Commun., vol. 5, pp. 23-27, Feb. 1998.

[27] F. Adachi and M. Sawahashi, "IMT-2000-challenges of wireless millennium," in Wireless Communication Technologies: New Multimedia Systems, N. Morinaga, S. Sampei, and R. Kohno, Eds. New York: Kluwer, 2000 , ch. 12 , pp. $263-293$.

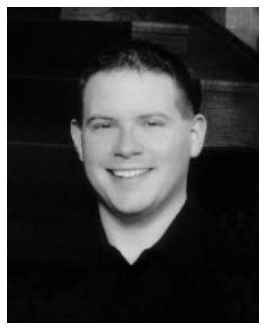

David J. Russell received the B.S. degree in computer engineering and the M.S. degree in electrical engineering from the University of Nebraska, Lincoln, in 1996 and 2001, respectively. He is currently working toward the Ph.D. degree in the Department of Electrical Engineering, University of Nebraska-Lincoln.

From 1996 to 1997, he was with Motorola Space and Systems Technology Group, Scottsdale, AZ, where he was a Software Engineer. Since 1997, he has been with EFJohnson, Lincoln, NE, where he is a Principal Development Engineer. His research interests include digital signal processing and wireless communications.

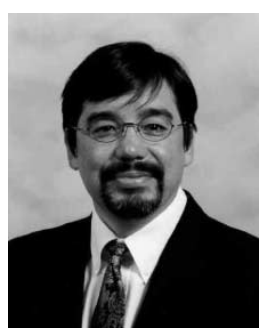

Robert D. Palmer (M'89-SM'93) received the $\mathrm{Ph} . \mathrm{D}$. degree in electrical engineering from the University of Oklahoma, Norman, in 1989. His Ph.D. studies focused on the application of advanced signal-processing techniques to atmospheric radar.

From 1989 to 1991, he was a Postdoctoral Fellow at the Radio Atmospheric Science Center, Kyoto University, Kyoto, Japan. After his stay in Japan, he held the position of Research Associate in the Physics Department, Clemson University, Clemson, SC. He joined the faculty of the Department of Electrical Engineering, University of Nebraska-Lincoln in January 1993 where he now holds the rank of Professor. He has published extensively in the general area of radar remote sensing of the atmosphere, with an emphasis on the use of multiple frequencies/receivers for interferometry and generalized imaging problems. 